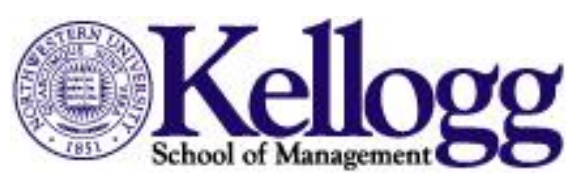

CMSEMS

Center for Mathematical Studies in Economics

And Management Science

Disc ussion Paper \#1542

\title{
On the Smoothness of Value Functions
}

\author{
Bruno Strulovici and Martin Szydlowski
}

Northwestem University

August 23, 2012

J EL Classific a tion: C61, D9, D83, D86, E20, G 11

Keywords: Optimal Control, Optimal Stopping, Smooth Pasting, Super Contact,

Comparative Statics, Envelope Theorem, Strong Solution, Markov Control, HJ B Equation
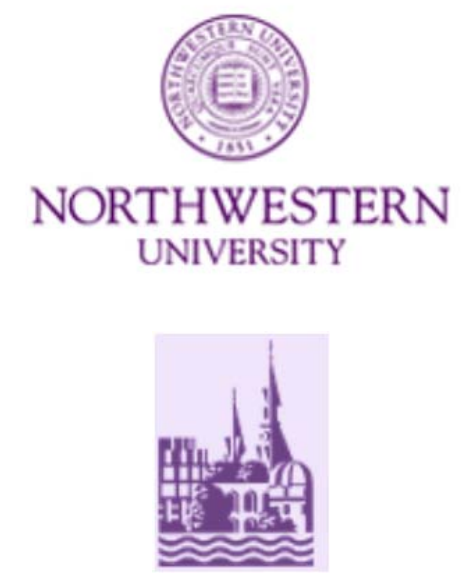


\title{
On the Smoothness of Value Functions and the Existence of Optimal Strategies*
}

\author{
Bruno Strulovici \\ Northwestern University
}

\author{
Martin Szydlowski \\ Northwestern University
}

August 23, 2012

\begin{abstract}
In dynamic models driven by diffusion processes, the smoothness of the value function plays a crucial role for characterizing properties of the solution. However, available methods to ensure such smoothness have limited applicability in economics, and economists have often relied on either model-specific arguments or explicit solutions. In this paper, we prove that the value function for the optimal control of any time-homogeneous, one-dimensional diffusion is twice continuously differentiable, under Lipschitz, growth, and non-vanishing volatility conditions. Under similar conditions, the value function of any optimal stopping problem is continuously differentiable. For the first problem, we provide sufficient conditions for the existence of an optimal control. The optimal control is Markovian and constructed from the Bellman equation. We also establish an envelope theorem for parameterized optimal stopping problems. Several applications are discussed, including growth, dynamic contracting, and experimentation models.
\end{abstract}

Keywords: Optimal Control, Optimal Stopping, Smooth Pasting, Super Contact, Comparative Statics, Envelope Theorem, Strong Solution, Markov Control, HJB Equation.

\section{Introduction}

Ever since Louis Bachelier formalized the concept of Brownian motion to study financial markets, diffusion processes have played an increasingly important role in economic analysis. Diffusions are used to model macroeconomic and financial shocks, incoming news in learning and experimentation

\footnotetext{
${ }^{*}$ We are grateful for comments from John Quah and Yuliy Sannikov. Part of this research was accomplished while the first author was visiting the Economic Theory Center at Princeton University, whose hospitality is gratefully acknowledged. This author is also thankful for financial support from the NSF under Grant No. 1151410.
} 
environments, and stochastic output and demand. Harnessing the relevant techniques has proved a challenge to economists, however, so much so that it has motivated a book by Dixit (1993) on the "art of smooth pasting," which included a heuristic justification for the differentiability of value functions at optimal stopping thresholds.

In dynamic models, the smoothness of value functions plays a crucial role to investigate the properties of optimal decisions and payoffs, as illustrated by the celebrated Benveniste-Scheinkman theorem. In growth models, for example, it is used with the Bellman (or, for diffusions, HamiltonJacobi-Bellman "HJB") equation to prove monotonicity of the optimal consumption and investment in the state and in parameters of the model. In dynamic contracting models, the agent's optimal effort, consumption, and continuation utility, and the principal's risk attitude can all be studied with the HJB equation, while an analytical solution is typically not attainable. In bandit problems, many properties of the optimal experimentation strategy can be characterized without an explicit solution, as we also illustrate.

In all these applications, the key is to show that the value function is smooth enough to satisfy the HJB equation. For the optimal control of diffusions, "smoothness" thus requires (and means in this paper) twice differentiability of the value function, and is sometimes known as the super contact condition 1 In pure stopping problems, "smoothness" requires (and means) that the value function is once differentiable, and is known as the smooth pasting condition.

Surprisingly, economists do not have available at hand a simple theorem guaranteeing such smoothness in diffusion models. The so-called "verification theorems" are used to check that a smooth candidate solution is equal to the value function, but do not provide this candidate solution, or guarantee its existence. In stopping problems, the usual arguments for smooth pasting are heuristic and typically assume that the optimal stopping region takes on a specific form. In optimal control problems, economists have used ad hoc proofs to establish that the value functions of particular models solved the HJB equation.

This paper provides two theorems for the smoothness of value functions, which hold under simple and easily checked conditions. Both theorems pertain to infinite horizon models with a onedimensional, time-homogeneous diffusion state, which are ubiquitous in economics. The first theorem states, under a non-vanishing volatility condition and standard continuity and linear growth conditions, that the value function of any optimal control problem is twice continuously differentiable and satisfies the HJB equation everywhere. The result holds for bounded as well as unbounded domains, and for any compact control space. In particular, we do not assume that

\footnotetext{
${ }^{1}$ The super contact condition has been extensively used to characterize optimal solutions. See, e.g., DeMarzo and Sannikov (2006) and DeMarzo et al. (2012).
} 
the control space is convex or one dimensional. This result relaxes a number of assumptions from earlier work, sometimes aimed at dealing with state spaces of arbitrary dimensions ${ }^{2}$ Our result also differs from the viscosity solution approach ${ }^{3}$ introduced by Crandall and Lions (1983) and Lions (1983), which is significantly less well known by economists and harder to work with.4

The second theorem states, under similar conditions, that the value function of any stopping problem is continuously differentiable, as a long as the terminal value function has this property $5^{5}$ For both theorems, the conditions we find were previously known to be necessary for the results $6^{6}$ but we are not aware of previous theorems showing that these conditions, taken together, are sufficient. $]^{7}$

A more subtle yet equally crucial issue concerns the existence of an optimal strategy. Indeed, just because one has proved that the value function of a given problem was smooth enough to solve the Bellman equation, this does not imply the existence of an optimal control, even if the set of maximizers of the equation is nonempty at all states. The key issue here is whether the corresponding controls generate a well defined trajectory for the state process. There are two concepts of solutions to stochastic differential equations: the strong one requires that the state trajectory is entirely determined by the control process and the modeled exogenous uncertainty,

\footnotetext{
${ }^{2}$ Twice differentiability of the value function has been studied by Krylov (1980), who assumes that the state space is finite and that the control space is convex. The existence of solutions to multidimensional boundary value problems, which include HJB equations with multidimensional states, has been studied by Noussair (1979), Evans (1983), and Safonov (1989). See also Gilbarg and Trudinger (2001). Methods for multidimensional problems are typically based on fixed-point arguments which require strong smoothness assumptions on the coefficients and payoffs, among other assumptions. Twice differentiability has also been studied for specific models in Operations Research (see, e.g., Harrison and Taksar (1983)).

${ }^{3}$ A related approach consists in finding weak (non classical) solutions in the relevant Sobolev space. Differentiability is then replaced by the concept of a weak derivative.

${ }^{4}$ Indeed, as economic theorists are aware, kinks in policies and value functions can play a crucial role in economic analysis, and it is important to determine when such kinks may occur and when they can be safely ruled out.

${ }^{5}$ The arguments can be extended to combining control and stopping problems. See Section 5.

${ }^{6}$ Touzi (2010) provides an example with an unbounded control space in which the value function is not differentiable. Øksendal and Sulem (2007, p.139) consider a pure stopping problem in which the terminal function is not differentiable, and show that the value function is not differentiable either at one of the stopping thresholds. With a vanishing volatility, the smoothing effect of the Brownian motion is absent at some states, and it is easy to build deterministic examples in which the value function is not twice differentiable.

${ }^{7}$ Recent results on the necessity of smooth pasting are provided by Dayanik and Karatzas (2003), Peškir and Shiryaev (2006), and Villeneuve (2007), using methods based on the scale function of the SDE, or by "excessivity" of the value function. Among other assumptions, one difference with our theorem is that these results focus on the case in which there is no flow payoff. The integral of the flow payoff can be converted into an additional state, reducing the problem to one without any flow payoff. Unfortunately, this conversion creates a multidimensional state space, in which some states have zero volatility. Peškir and Shiryaev (2006) propose a method for proving the necessity of smooth pasting which, they mention, can be extended to multidimensional states. However, that method requires a minimal volatility condition that is not satisfied by the converted state.
} 
a property that always holds in discrete stochastic optimization. The weak one admits that the trajectory also depends on some other source of uncertainty beyond the one explicitly modeled. Roughly put, it means that the model can be extended by adding enough exogenous uncertainty, on which the state can depend (beyond the initial Brownian motion), so that the equation has a well-defined solution. This second concept is counter-intuitive at best. As preeminent control theorists Yong and Zhou (1999, p. 64) have put it "the strong formulation is the one that stems from the practical world, whereas the weak formulation sometimes serves as an auxiliary but effective mathematical model aiming at ultimately solving problems with the strong formulation."

In economic models with a controlled diffusion, there are currently few options, other than finding an explicit solution, for guaranteeing the existence of an optimal control 8 To see the difficulty, consider the simple case of a binary control (such as a two-armed bandit problem). In such case, any nontrivial Markovian control has to "jump" as the diffusion hits some regions of the state space, creating discontinuities in the coefficients generating the stochastic equation for the state. In particular, the usual Lipschitz continuity condition for the existence of a solution is violated, since the coefficient is not even continuous.

Fortunately, there exist specific results for the case of one-dimensional diffusions, establishing the existence of a unique strong solution as long as the volatility coefficient has bounded variation.9 Building on these results, we identify conditions under which an optimal control exists, which are all based on the HJB equation, and hence all make use of the first theorem that we have established. Proving the existence of optimal strategies is therefore a first application of the smoothness result.

Some of our existence results are based on establishing, via an envelope theorem, differentiability of the control, and are illustrated in several applications. Our other existence results are based on the theory of monotone comparative statics: if one can show that the control constructed from the HJB equation is monotonic, or at least piecewise monotonic, this will guarantee that it has bounded variation and, under a simple regularity condition, that the volatility has bounded variation. Proving such monotonicity is nontrivial, because the value function and its derivatives can typically not be computed explicitly. However, it is precisely for this kind of situations that the theory of comparative statics has been developed. We provide several applications illustrating this connection.

\footnotetext{
${ }^{8}$ Stroock and Varadhan (1979) and Krylov (1980) developed results showing the existence of weak solutions. For more general existence results with weak solutions, see the survey by Borkar (2005). It is also well known, and shown in this paper (Section 3), that there always exist $\varepsilon$-optimal controls that generate strong solutions. Fleming and Soner (1993) assume that the control is Lipschitz in the state (and jointly continuous in the time-state space, when it also depends explicitly on time).

${ }^{9}$ The first such result is due to Nakao (1972). See Section 4
} 
Economists have long been interested in another kind of smoothness and comparative statics: describing how the value function of an optimization problem varies with some parameters of the problem. We provide several such results for optimal stopping problems, building on arguments used in earlier sections, in which the parameter can enter the flow and terminal payoff functions as well as the drift and volatility of the diffusion. First, we impose a strict single crossing condition guaranteeing that the continuation and stopping regions are separated by a unique threshold. This uniqueness is combined with the insights of Milgrom and Segal (2002) to provide an envelope theorem for the value function: the value function is smooth in the parameter, and its derivative is equal to the derivative of the expected payoff with respect to the parameter, holding the stopping threshold fixed. Finally, this envelope theorem is combined with a standard argument to derive a representation for the derivative of the value function with respect to the parameter, based on similar derivatives for all the primitives of the problem. This yields a "dynamic sensitivity equation," which may be used to prove other properties of the value function, as we illustrate 10

Beyond these results, it is important to understand why the value function is smooth. What is it in the optimal control or stopping strategy that makes the expected payoff smooth in the state, whereas it is not so for arbitrary suboptimal strategies? Whereas heuristic arguments are well known for smooth pasting, the answer is less obvious for optimal control problems 11 In Section 3 we explain exactly where optimality of the control plays a role: it guarantees, by an application of Berge's Maximum Theorem, that the maximized objective of the HJB equation is continuous in the state and, hence, that usual techniques for proving the existence of a solution to nonlinear elliptic second-order equations apply 12

For the optimal stopping problem, the argument applies to problems with arbitrary stopping and continuation regions. The key observation is that the continuation region always consists of disjoint open intervals over which the value function solves a standard boundary value problem, and that a local argument can be applied to the endpoints of such interval to guarantee smoothness at those points. The local argument works by contradiction and is based on a standard "fishing" technique: if the value function is not differentiable at some stopping point, one may raise the slope of some

\footnotetext{
${ }^{10}$ This equation is well known when there is no stopping problem. For an application in finance, see DeMarzo and Sannikov (2006).

${ }^{11}$ Dumas (1991), who introduced the concept of a "super contact condition" to study twice differentiability, provides useful intuition for the result.

${ }^{12}$ There is another particularity of our approach: in much of the analysis, we need to work with solutions to boundary value problems (BVP), in which the terminal conditions of the equation are matching values at both endpoints of the problem, rather than initial value problems (IVP), which match value and derivative at a single endpoint. To move from IVP to BVP, we exploit the fact that the Hamiltonian is nondecreasing in the value function (Condition 2), which yields a tailored argument and gets rid of a more demanding regularity assumption on the primitives.
} 
initial value problem to get a higher payoff 13

The results are illustrated with several applications. The first one concerns an optimal growth problem. Relaxing usual concavity and convexity assumptions, we show that the value function is always smooth and solves the Bellman equation under standard regularity conditions. We also discuss conditions for the existence of an optimal control. Our second application concerns multiarmed bandit problems with a common state. Each arm is a choice that is informative about the true state of the world, and has a state-dependent payoff. The value function is, without any further assumptions, always twice differentiable. When the number of arms is finite, the number of switching points between arms is shown to be finite (and uniformly bounded), although it may be strictly optimal to use a given arm over disjoint intervals of beliefs. We also provide general conditions for the existence of an optimal control when the agent can also allocate resources across two arms, rather than merely choose one of them. The third application revisits the principalagent model analyzed by Sannikov (2008), proving the existence of an optimal contract under a simple condition on the effort cost function 14 We also provide, as a simple illustration of our results, a general proof that the option value of waiting in optimal stopping problems is positive and increasing in the volatility of the underlying process.

The paper is organized as follows. Section 2 introduces the general optimal control problem. Section 3 proves that the value function is twice continuously differentiable. Section 4 provides sufficient conditions for the existence of an optimal control. Section 5 turns to the optimal stopping problem, proving the continuous differentiability of the value function. Section 6 considers a large class of parametric optimal stopping problems, in which the optimal threshold is shown to be unique, and provides an envelope theorem and a dynamic equation for the derivative of the value function with respect to the parameter. Sections 36 include examples illustrating their respective results. Section 7 concludes. Technical proofs are in the Appendix.

\section{Control Problem}

We are given a filtered probability space $\left(\Omega, \mathcal{F},\left\{\mathcal{F}_{t}\right\}_{t \in \mathbb{R}_{+}}, \mathbb{P}\right)$ which satisfies the usual conditions and whose outcomes are identified with the paths of a standard Brownian motion, denoted by $B \longdiv { 1 5 }$

\footnotetext{
${ }^{13}$ To conclude the argument, one must also show that the modified solution is equal to the expected payoff achieved under some stopping policy. Another subtlety is that a stopping point could be an accumulation point of an infinitely alternating pattern of stopping and continuation regions, which become smaller as they close to the point. One has to show that the derivative is well defined and continuous at such point.

${ }^{14}$ Without this condition, the continuation utility process derived for the contract characterized in that paper is only guaranteed to exist in the sense of a weak solution.

${ }^{15}$ We refer the reader to Karatzas and Shreve (1998) for the standard concepts used in this paper.
} 
We consider a process $\left\{X_{t}\right\}_{t \in \mathbb{R}_{+}}$controlled by another process $\left\{A_{t}\right\}_{t \in \mathbb{R}_{+}}$, taking values in a nonempty closed interval $\mathcal{X}$ of $\mathbb{R}$ with (possibly infinite) endpoints $\underline{x}<\bar{x}$, and following the dynamic equation

$$
\begin{aligned}
d X_{t} & =\mu\left(X_{t}, A_{t}\right) d t+\sigma\left(X_{t}, A_{t}\right) d B_{t} \\
x_{0} & =x
\end{aligned}
$$

Assumption 1 There exists a nonempty compact metric space $\mathcal{K}$ such that $A_{t} \in \mathcal{K}$ for all $t .16$

A control process is said to be admissible if it is adapted to the filtration $\left\{\mathcal{F}_{t}\right\}_{t \in \mathbb{R}_{+}}$, satisfies Assumption 1, and the Equation (1) has a unique strong solution ${ }^{17}$ The set of admissible control processes is denoted by $\mathcal{A}$.

Given an admissible control $A$, the agent's expected payoff is given by

$$
v(x, A)=E\left[\int_{0}^{\kappa} e^{-r t} f\left(X_{t}^{A}, A_{t}\right) d t+e^{-r \kappa} g\left(X_{\kappa}^{A}\right)\right],
$$

where $X_{t}^{A}$ is the process starting at $x$ and controlled by $A, f\left(X_{t}^{A}, A_{t}\right)$ is the flow payoff at time $t$, $\kappa=\inf \left\{t: X_{t} \notin(\underline{x}, \bar{x})\right\}$ is the first time at which the boundary of $\mathcal{X}$ is hit, and $g(\underline{x})$ and $g(\bar{x})$ are given constants (relevant only when their argument is finite). Assumptions 2 and 3 , stated shortly, guarantee that the expected payoff is well defined for any admissible control (see Lemma 1 below). The (optimal) value function ${ }^{18}$ of the problem starting at $x$, denoted $v(x)$, is defined by

$$
v(x)=\sup _{A \in \mathcal{A}} v(x, A) .
$$

An admissible control is said to be optimal if $v(x, A)=v(x)$.

Assumption 2 There exists $K>0$ such that, for all $\left(x, x^{\prime}, a\right) \in \mathcal{X}^{2} \times \mathcal{K}$,

$$
\left|\mu(x, a)-\mu\left(x^{\prime}, a\right)\right|+\left|\sigma(x, a)-\sigma\left(x^{\prime}, a\right)\right|+\left|f(x, a)-f\left(x^{\prime}, a\right)\right| \leq K\left|x-x^{\prime}\right|,
$$

and the functions $\mu(x, \cdot), \sigma(x, \cdot), f(x, \cdot)$ are continuous in $a$, for each $x \sqrt{19}$

The last assumption contains several bounds on the primitives: standard linear growth conditions, a uniform lower bound on $\sigma$, and a condition guaranteeing that, for any control, the state grows at a rate slower than the discount rate.

\footnotetext{
${ }^{16}$ The assumption that $\mathcal{K}$ is independent of $x$ is only for expositional simplicity. The analysis can be extended to the case in which the control set depends on $x$, provided that i) for each $x, \mathcal{K}(x)$ is a nonempty, closed subset of the compact metric space $\mathcal{K}$, and ii) the correspondence $x \mapsto K(x)$ is continuous. See Footnote 26 .

${ }^{17}$ This definition of admissibility is the one used, among others, by the classic control theory textbook of Fleming and Soner (1993). See also Pham's (2009) recent textbook.

${ }^{18}$ To avoid confusion, we reserve the expression "value function" to the optimal expected payoff, and use the expression "expected payoff" when the control is arbitrary.

${ }^{19}$ When $\mathcal{K}$ is a finite set, this latter assumption is vacuous.
} 
Assumption 3 There exist constants $K_{1}^{\mu}, K_{2}^{\mu}, K^{\sigma}, K^{f}$, and $\underline{\sigma}$ such that ${ }^{20} K_{2}^{\mu}<r$ and $0<\underline{\sigma} \leq$ $|\sigma(x, a)|,|\mu(x, a)| \leq K_{1}^{\mu}+K_{2}^{\mu}|x|,|\sigma(x, a)| \leq K^{\sigma}(1+|x|)$, and $|f(x, a)| \leq K^{f}(1+|x|)$ for all $(x, a) \in \mathcal{X} \times \mathcal{K}$.

\section{Twice Differentiability of the Value Function}

Our objective is to prove that the value function is twice differentiable in the interior of $\mathcal{X}$ and solves the Hamilton-Jacobi-Bellman (HJB) equation

$$
0=\sup _{a \in \mathcal{K}}-r v(x)+f(x, a)+\mu(x, a) v^{\prime}(x)+\frac{1}{2} \sigma^{2}(x, a) v^{\prime \prime}(x)
$$

with given boundary conditions

$$
v(\underline{x})=g(\underline{x}) \text { if } \underline{x} \text { is finite, and } v(\bar{x})=g(\bar{x}) \text { if } \bar{x} \text { is finite. }
$$

TheOREm 1 Under Assumptions 1 [3, the following holds:

i) The value function is finite and has linear growth: $|v(x)| \leq K_{v}(1+|x|)$ for some positive constant $K_{v}$.

ii) The HJB equation has a twice continuously differentiable solution.

iii) Any solution to the HJB equation that has linear growth is equal to the value function.

Finiteness is only an issue when $\mathcal{X}$ is unbounded, and follows from the following lemma, proved in the Appendix.

LEMma 1 For any admissible control $A$, initial condition $x$, and $\tilde{r}>K_{2}^{\mu}, \lim _{t \rightarrow+\infty} E\left|X_{t}^{A}\right| e^{-\tilde{r} t}=0$ and $E\left[\int_{0}^{\infty} e^{-\tilde{r} t} f\left(X_{t}^{A}, A_{t}\right) d s\right]<+\infty$.

This lemma, combined with the linear growth condition on $f$ and the fact that $K_{2}^{\mu}<r$ immediately implies that $v$ is finite and satisfies a linear growth condition 21

The rest of the proof consists of the following steps: 1) Prove the existence of a solution, $w$, to the HJB equation, which has linear growth; 2) Construct a control process based on this solution; 3)

\footnotetext{
${ }^{20}$ The condition $K_{2}^{\mu}<r$ can be dropped if $f$ is bounded. In that case, the control problem is equivalent to one in which $X$ is replaced by a smooth increasing transformation $Y$ of $X$, that satisfies this condition, without affecting other conditions.

${ }^{21}$ Precisely, Lemma 1 and the growth condition on $f$ guarantee that $f\left(X_{t}, A_{t}\right)$ grows at most at rate $K_{2}^{\mu}<$ $r$. This implies that the integral payoff is bounded linearly in $x$. The terminal payoff is bounded above by $\max \left\{g(\underline{x}) 1_{\underline{x}>-\infty}, g(\bar{x}) 1_{\bar{x}<+\infty}\right\}$, which is also finite.
} 
Prove that the solution is the value function of the problem, and that either the control constructed in 2) is optimal, or that it can be approximated by a sequence of admissible controls.

These steps will imply that any solution of the HJB equation with linear growth must coincide with the value function of the problem and, therefore, will show the uniqueness claimed in Part iii) of Theorem 1 .

We first show the existence of a solution to the HJB equation. This result follows from Proposition 1 below, which is proved in the Appendix. The proposition relies on the following conditions for an arbitrary function $\bar{H}(x, p, q)$ defined on $\mathcal{X} \times \mathbb{R}^{2}$.

COndition 1 On any compact interval $\mathcal{X}_{0}$ of $\mathcal{X}$, there exist constants $M$ and $K$ such that

i) $|\bar{H}(x, p, q)| \leq M(1+|p|+|q|)$,

ii) $|\bar{H}(x, p, q)-\bar{H}(x, \tilde{p}, \tilde{q})| \leq K(|p-\tilde{p}|+|q-\tilde{q}|)$,

iii) $\bar{H}$ is continuous in $x$ for each $(p, q)$.

Condition 2 For all $(x, q), \bar{H}(x, \cdot, q)$ is nonincreasing in $p$.

Condition 3 For each $\bar{K}>0$, there exist $K_{1}, K_{2}>\bar{K}$ such that for all $x \in \mathcal{X}$, and $\varepsilon \in\{-1,1\}$,

$$
\left.\bar{H}\left(x, K_{1}+K_{2}|x|, \varepsilon K_{2}\right)<0 \text { and } \bar{H}\left(x,-K_{1}-K_{2}|x|\right), \varepsilon K_{2}\right)>0 .
$$

Proposition 1 Suppose that $\bar{H}$ satisfies Conditions 1 3. Then, for any finite $\underline{v}$ and $\bar{v}$, there exists a twice continuously differentiable solution to the equation

$$
w^{\prime \prime}+\bar{H}\left(x, w, w^{\prime}\right)=0
$$

which satisfies $w(\underline{x})=\underline{v}$ if $\underline{x}$ is finite, and $w(\bar{x})=\bar{v}$ if $\bar{x}$ is finite. Moreover, there exists a positive constant $K_{v}$ such that

$$
|w(x)| \leq K_{v}(1+|x|) \text { for all } x \in \mathcal{X} .
$$

We apply Proposition 1 to Equation (3) by checking Conditions 1 - 3 .

Proposition 2 Under Assumptions 1 3, the HJB equation (3) has a twice continuously differentiable solution $w$ on $\mathcal{X}$, which has linear growth.

\footnotetext{
${ }^{22}$ Parts ii) and iii) imply that $H$ is jointly continuous in $(x, p, q)$. Although we do not use this fact explicitly in the proof, it explains why the second derivative of the solution is also continuous.
} 
Proof. Equation (3) can be rewritten as

$$
w^{\prime \prime}+H\left(x, w, w^{\prime}\right)=0
$$

where23

$$
H(x, p, q)=\max _{a \in \mathcal{K}} \frac{2}{\sigma^{2}(x, a)}(-r p+f(x, a)+\mu(x, a) q) .
$$

We show that $H$ satisfies Conditions 1, 2, and 3 .

Let

$$
h(a, x, p, q)=\frac{2}{\sigma^{2}(x, a)}(-r p+f(x, a)+\mu(x, a) q),
$$

so that $H(x, p, q)=\max _{a \in \mathcal{K}} h(a, x, p, q)$. Assumptions $1-3$ guarantee that $h$ is continuous in $a$ and Lipschitz in $x$, uniformly in $a$. Because $r$ and $\sigma^{2}$ are positive, $h$ is decreasing in $p$ and Condition 2 is satisfied.

To verify Condition 1, we use the following inequality ${ }^{24}$

$$
|H(x, p, q)-H(x, \tilde{p}, \tilde{q})| \leq \max _{a \in \mathcal{K}} \frac{2}{\sigma^{2}(x, a)}|(-r p+f(x, a)+\mu(x, a) q)-(-r \tilde{p}+f(x, a)+\mu(x, a) \tilde{q})| .
$$

This implies that

$$
|H(x, p, q)-H(x, \tilde{p}, \tilde{q})| \leq \frac{2}{\underline{\sigma}^{2}}\left(r|p-\tilde{p}|+\left(K_{1}^{\mu}+K_{2}^{\mu}|x|\right)|q-\tilde{q}|\right),
$$

and proves the Lipschitz condition for any compact interval $\mathcal{X}_{0}$ of $\mathcal{X}$. Similarly, the growth condition follows because $\mu$ and $f$ are bounded on any compact support and $\sigma^{2}$ is bounded below by $\underline{\sigma}^{2}>0$. Continuity of $H$ in $x$, the last part of Condition 1 is the key to guarantee that the value function is twice differentiable, even when the optimal control jumps. It is due to Berge's Maximum Theorem. Because the objective $h$ is continuous in $a$ and Lipschitz in $x$, uniformly in $a$, it is easy to show that $h$ is jointly continuous in $(x, a){ }^{25}$ Since also the action set $\mathcal{K}$ is compact, the Maximum Theorem applies, which proves that $H$ is continuous in $x{ }^{26}$

\footnotetext{
${ }^{23}$ Because all functions are continuous in $a, \mathcal{K}$ is compact, and $\sigma$ is bounded below away from zero, the supremum is achieved as a maximum.

${ }^{24}$ More generally, if $H(\theta)=\max _{a \in \mathcal{K}} h(a, \theta)$, one can prove that $|H(\theta)-H(\tilde{\theta})| \leq \max _{a \in \mathcal{K}}|h(a, \theta)-h(a, \tilde{\theta})|$. For example, suppose that $a, \tilde{a}$ maximize $h$ at $\theta$ and $\tilde{\theta}$, respectively. Then, $H(\theta)-H(\tilde{\theta})=h(a, \theta)-h(\tilde{a}, \tilde{\theta}) \leq h(a, \theta)-$ $h(a, \tilde{\theta}) \leq \max _{a \in \mathcal{K}}|h(a, \theta)-h(a, \tilde{\theta})|$. The other inequality is proved similarly.

${ }^{25}$ This is shown as follows. We fix and omit from the notation some values for $p$ and $q$, and suppose that $\left(a_{n}, x_{n}\right)$ converges to $(a, x)$. We have $\left|h(x, a)-h\left(x_{n}, a_{n}\right)\right| \leq\left|h(x, a)-h\left(x, a_{n}\right)\right|+\left|h\left(x, a_{n}\right)-h\left(x_{n}, a_{n}\right)\right|$. The first term converges to zero by continuity of $h$ with respect to $a$, while the second term converges to zero because $h$ is Lipschitz in $x$, uniformly in $a$.

${ }^{26}$ Berge's Theorem also applies if the control domain $\mathcal{K}(x)$ depends on $x$ and satisfies the conditions provided in Footnote 16
} 
There remains to verify that Condition 3 holds. $H\left(x, K_{1}+K_{2}|x|, \varepsilon K_{2}\right)$ is negative whenever

$$
-r\left(K_{1}+K_{2}|x|\right)+K^{f}(1+|x|)+\left(K_{1}^{\mu}+K_{2}^{\mu}|x|\right) K_{2}<0 .
$$

Since $K_{2}^{\mu}<r$, this inequality holds for all $x \in \mathbb{R}$ (and hence, on $\mathcal{X}$ ) if and only if

$$
K_{2} \geq \frac{K^{f}}{r-K_{2}^{\mu}} \quad \text { and } \quad K_{1}>\frac{K^{f}+K_{1}^{\mu} K_{2}}{r}
$$

Thus the assumptions in Proposition 1 are satisfied, which shows existence of a solution to the HJB equation for arbitrary boundary conditions at $\underline{x}$ and $\bar{x}$, whenever these points are finite.

What role does optimization play for smoothness? As mentioned above, continuity of $H$ in $x$ is required to guarantee that the value function is twice differentiable, and is due to an application of Berge's Maximum Theorem to the maximization of the objective $h$. If, instead of a maximum selection $x \mapsto \hat{a}(x)$ of $h$, we had chosen an arbitrary selection $x \mapsto \tilde{a}(x)$, the resulting function $\tilde{H}(x, p, q)=h(\tilde{a}(x), x, p, q)$ would generally not be continuous in $x$. This explains why the expected payoff is twice differentiable for the optimal control, whereas it may fail to be so for a strictly suboptimal control: recall that the second derivative is given by $v^{\prime \prime}(x)=-H\left(x, v(x), v^{\prime}(x)\right)$. If $H$ is discontinuous in $x$, the value function cannot be twice continuously differentiable, or even twice differentiable there, since the left and right second derivatives at $x$ will be different.

Proof that the candidate solution $w$ is equal to the value function $v$ We split up the proof into two inequalities.

Lemma 2 Let $w$ be a solution to the HJB equation (3) that has linear growth, and let $v(x, A)$ be the expected value function given any admissible control $A$. Then, $w(x) \geq v(x, A)$ for all $x \in \mathcal{X}$.

Proof. The proof follows a standard verification argument, which is included here for completeness. For any fixed, admissible control $A$ and finite time $T$, Itô's formula implies, for the diffusion $X^{A}$ controlled by $A$ and starting at $x$, that

$$
\begin{array}{r}
e^{-r(T \wedge \kappa)} w\left(X_{T \wedge \kappa}^{A}\right)=w(x)+\int_{0}^{T \wedge \kappa} e^{-r t}\left(-r w\left(X_{t}^{A}\right)+\mu\left(X_{t}^{A}, A_{t}\right) w^{\prime}\left(X_{t}^{A}\right)+\frac{1}{2} \sigma^{2}\left(X_{t}^{A}, A_{t}\right) w^{\prime \prime}\left(X_{t}^{A}\right)\right) d t \\
+\int_{0}^{T \wedge \kappa} e^{-r t} \sigma\left(X_{t}^{A}, A_{t}\right) d B_{t}, \quad(8)
\end{array}
$$

where the stopping time $T \wedge \kappa=\min \{T, \kappa\}$ guarantees that $X_{t}^{A}$ has not yet hit the boundary of $\mathcal{X}$ on time interval over which Itô's lemma is applied. The term $e^{-r t} \sigma\left(X_{t}^{A}, A_{t}\right)$ is square integrable over $[0, T]$ and the stochastic integral has zero mean (see Section $\mathrm{A}$ ). Taking expectations and using (3), we get the inequality

$$
E\left[\int_{0}^{T \wedge \kappa} e^{-r t} f\left(X_{t}^{A}, A_{t}\right) d t\right] \leq w(x)-E\left[e^{-r(T \wedge \kappa)} w\left(X_{T \wedge \kappa}^{A}\right)\right] .
$$


The linear growth condition satisfied by $w$, along with Lemma 1, guarantees that, on the event $\kappa=+\infty$, we have $\lim _{T \rightarrow \infty} E\left[e^{-r T} w\left(X_{T}^{A}\right)\right]=0$. Taking the limit of $(9)$ as $T$ goes to infinity 27 and using the equality $w\left(X_{\kappa}^{A}\right)=g\left(X_{\kappa}^{A}\right)$ yields $v(x, A) \leq w(x)$.

For the reverse inequality, we first obtain a candidate optimal control $A_{t}^{*}$ from the solution to the HJB equation $w$. This candidate need not be admissible, because the stochastic differential equation (1) may fail to have a unique strong solution. We will use a result by Nakao (1972), who has shown ${ }^{28}$ that a one-dimensional SDE has a unique strong solution if its drift is measurable and its volatility has bounded variation and is bounded away from zero. 29 We exploit this property to construct an approximation to the candidate control which is admissible and gets arbitrarily close to the desired inequality.

Lemma 3 Let $w$ be a solution to the HJB equation (3) that has linear growth. Then $w(x) \leq v(x)$ for all $x \in \mathcal{X}$.

Proof. We construct a candidate optimal control as follows. Take a solution to the HJB equation $w$, and define $\mathcal{M}(x) \subset \mathcal{K}$ as the set of maximizers of the equation

$$
r w(x)=\max _{a \in \mathcal{K}} f(x, a)+\mu(x, a) w^{\prime}(x)+\frac{1}{2} \sigma(x, a)^{2} w^{\prime \prime}(x) .
$$

The objective is continuous in $a$ and in $x$, and $\mathcal{K}$ is nonempty and compact. The measurable maximum theorem ${ }^{30}$ thus guarantees the existence of a measurable selection $\hat{a}(x) \in \mathcal{M}(x)$ of maximizers.

If the control $A_{t}^{*}=\hat{a}\left(X_{t}\right)$ is admissible, applying the previous verification argument, this time with an equality, shows that $w(x)=v\left(x, A^{*}\right)$ and, hence, $w(x) \leq v(x)$. In general, the volatility function $\hat{\sigma}(x)=\sigma(x, \hat{a}(x))$ can jump, violating the standard Lipschitz (or Hölder) continuity conditions usually assumed for the existence of a strong solution and, hence, for admissibility of the control 31 We circumvent this issue by the following approximation argument.

Fix any $\varepsilon>0$ and consider a grid of $\mathcal{X}$ with equally spaced intervals of length $\eta$, to be chosen shortly. We define the Markovian control $\tilde{a}$ by $\tilde{a}(x)=\hat{a}(\chi(x))$ where $\chi(x)$ is the element of the

\footnotetext{
${ }^{27}$ The expectation of the integral converges, since the integrand goes to zero at a geometric rate, by Lemma 1

${ }^{28}$ More precisely, Nakao established pathwise uniqueness of weak solutions. This, combined with a major result due to Yamada and Watanabe (1971), shows the existence of a (unique) strong solution. See also Veretennikov (1981) and Revuz and Yor (2001, p. 392) for related results.

${ }^{29}$ Drift and volatility must also be bounded, which holds here for any compact interval of $\mathcal{X}$.

${ }^{30}$ See Aliprantis and Border (1999), p. 570.

${ }^{31}$ See, e.g., Karatzas and Shreve (1998), Chapter 5.2, Theorem 2.5.
} 
grid closest to $x{ }^{32}$ By construction, $\tilde{a}$ is piecewise constant. By Nakao (1972), the SDE

$$
d X_{t}=\mu\left(X_{t}, \tilde{a}\left(X_{t}\right)\right) d t+\sigma\left(X_{t}, \tilde{a}\left(X_{t}\right)\right) d B_{t}
$$

has a unique strong solution, because the function $x \mapsto \sigma(x, \tilde{a}(x))$ has bounded variation. Letting $\left\{\tilde{X}_{t}\right\}_{t \in \mathbb{R}_{+}}$denote this solution, the control $\left\{\tilde{A}_{t}\right\}_{t \in \mathbb{R}_{+}}$defined by $\tilde{A}_{t}=\tilde{a}\left(\tilde{X}_{t}\right)$ is admissible. If $\mathcal{X}$ is compact, the function $f(x, a)+\mu(x, a) w^{\prime}(x)+\frac{1}{2} \sigma(x, a)^{2} w^{\prime \prime}(x)-r w(x)$ is uniformly continuous in $(x, a)$ on $\mathcal{X} \times \mathcal{K}$. Therefore, we can choose a grid mesh $\eta$ small enough to guarantee that

$$
\begin{aligned}
& f(x, \tilde{a}(x))+\mu(x, \tilde{a}(x)) w^{\prime}(x)+\frac{1}{2} \sigma(x, \tilde{a}(x))^{2} w^{\prime \prime}(x)-r w(x) \\
& \geq f(\chi(x), \tilde{a}(x))+\mu(\chi(x), \tilde{a}(x)) w^{\prime}(\chi(x))+\frac{1}{2} \sigma(\chi(x), \tilde{a}(x))^{2} w^{\prime \prime}(\chi(x))-r w(\chi(x))-\varepsilon \geq-\varepsilon .
\end{aligned}
$$

Plugging this this inequality in the verification argument based on (8), yields $v(x, \tilde{A}) \geq w(x)-\varepsilon / r$. Since $v(x) \geq v(x, \tilde{A})$, taking the limit as $\varepsilon$ goes to zero yields the desired inequality $v(x) \geq w(x)$.

We now show the result if $\mathcal{X}$ is unbounded, focusing on the case in which $\mathcal{X}=\mathbb{R}$ (the case in which $\mathcal{X}$ is a half-line is treated similarly). To each $\mathcal{X}_{n}=[-n, n]$ corresponds a modulus of continuity $\eta_{n}$ such that (12) holds on $\mathcal{X}_{n}$ if the grid has mesh $\eta_{n}$ and $\tilde{a}$ is constructed as before. We construct $\tilde{a}$ on $\mathcal{X}_{1}$ by using the grid with mesh $\eta_{1}$ on that domain, then extend it on $\mathcal{X}_{2} \backslash \mathcal{X}_{1}$ by using the grid with mesh $\eta_{2}$ on that domain, and so on. This construction defines $\tilde{a}$ over $\mathbb{R}$. Moreover, $\tilde{a}$ is piecewise constant with finitely many jumps over any $\mathcal{X}_{n}$, and hence has bounded variation over any compact interval of $\mathbb{R}$. Finally, 12 holds, by construction, on the entire domain $\mathbb{R}$. The rest of the argument is unchanged.

\subsection{Example: Optimal Growth}

The analysis of growth models typically relies on some smoothness of the value function, as the success of the Benveniste-Scheinkman Theorem (1979) illustrates. When time is continuous, smoothness takes on a particularly important role: it guarantees that the Bellman equation is satisfied everywhere, and, hence, that it can be directly used to derive properties of optimal policies ${ }^{33}$ In models without uncertainty, concavity assumptions are often required to establish differentiability (see Benveniste-Scheinkman (1979) and, more recently, Rincon-Zapatero and Santos (2009, 2010)). Theorem 1 shows that such assumptions can be dispensed with when uncertainty is modeled by Brownian noise.

\footnotetext{
${ }^{32}$ We can adopt any arbitrary convention when there are two points of the grid that are closest to $x$.

${ }^{33}$ One illustration is provided by Quah and Strulovici (2012b), who show monotonicity of the capital growth in the discount factor. Their analysis connects supermodularity properties of the objective function in the HJB equation.
} 
Consider an agent with initial capital $x$ who seeks to maximize his lifetime utility by managing his capital through consumption, savings, and effort. Formally, the agent solves

$$
\sup _{A \in \mathcal{A}} E\left[\int_{0}^{\infty} e^{-r s} u\left(X_{s}^{A}, A_{s}\right) d s\right]
$$

subject to

$$
d X_{t}^{A}=\mu\left(X_{t}^{A}, A_{t}\right) d t+\sigma\left(X_{t}^{A}, A_{t}\right) d B_{t},
$$

where the control domain $\mathcal{K}$ is a compact subset of $\mathbb{R}^{k}$ for some $k \geq 1$, and the drift and volatility functions $\mu$ and $\sigma$ are such that $X$ is always nonnegative and that 0 is an absorption point, with $u(0, a)=\mu(0, a)=\sigma(0, a)=0$ for all $a$. The dimensions of $A$ can represent consumption, leisure and effort, technological choices, etc. $X_{t}^{A}$ is the capital available to the agent at time $t$.

We do not place any other restriction on the agent's technology and utility functions apart from the Lipschitz and linear growth conditions required by Theorem 1. By assuming that, for each $\varepsilon>0, \sigma(x, a)$ is uniformly bounded away from zero on $[\varepsilon,+\infty) \times \mathcal{K}$, we can apply ${ }^{34}$ Theorem 1 to show that the value function is $C^{2}$ on $(\varepsilon,+\infty)$ for each $\varepsilon>0$ and, hence, on the interior $(0,+\infty)$ of the entire domain. Moreover, it solves for all $x>0$ the HJB equation

$$
r v(x)=\max _{a \in \mathcal{K}} u(x, a)+\mu(x, a) v^{\prime}(x)+\frac{1}{2} \sigma^{2}(x, a) v^{\prime \prime}(x) .
$$

A major issue in such environment is to establish the existence of an optimal strategy for the agent. Section 4 provides general results which may be used in this setting. If the control $A$ consists only of a consumption choice, then the volatility $\sigma$ is typically independent of $X$ : the agent's consumption affects the dynamics of the capital $X$ only by reducing its drift. In that case, Corollary 1 of Section 4 implies that any measurable maximizing selection $a(x)$ of $(13)$ defines an admissible, optimal control. Section 4.1 analyzes existence for a more general control process.

\subsection{Example: Multi-Armed Bandit}

Consider a multi-armed bandit problem with a common unknown parameter $\tilde{\theta} \in\left\{\theta_{L}, \theta_{H}\right\}$, representing a binary, payoff relevant state of the economy. The agent must choose at each time an arm $i$ in some compact set $\mathcal{K}$, given his belief $X_{t} \in[0,1]=\operatorname{Pr}\left(\tilde{\theta}=\theta_{H} \mid \mathcal{F}_{t}\right)$ about $\tilde{\theta}$. Given the choice $A_{t}$ of an arm at time $t$, the agent learns about $\tilde{\theta}$ according to some equation 35

$$
d X_{t}^{A}=\mu\left(X_{t}^{A}, A_{t}\right) d t+\sigma\left(X_{t}^{A}, A_{t}\right) d B_{t} .
$$

\footnotetext{
${ }^{34}$ The termination value $g(\varepsilon)$ in $\sqrt{2}$ is set to the actual value function $v(\varepsilon)$, so that the value function on $[\varepsilon,+\infty)$ coincides, on that domain, with the value function of the initial control problem over the entire domain $[0,+\infty)$.

${ }^{35}$ Unlike a setting in which arms are independent, such as the one analyzed by Gittins (1979), here all arms relate to the same state. Analyzing control problems with multidimensional states is beyond the scope of this paper.
} 
Because the belief is a martingale, $\mu$ is identically equal to zero. Moreover, from standard computations (see, e.g., Bolton and Harris (1999)), the volatility has the form $\sigma\left(X_{t}, A_{t}\right)=X_{t}\left(1-X_{t}\right) \xi\left(A_{t}\right)$ and is thus entirely characterized by the vector $\{\xi(i)\}_{i \in \mathcal{K}} . \xi(i)$ is the signal-to-noise ratio of the aggregate signal generated by $\operatorname{arm} i$. The expected flow payoff from playing arm $i$ at time $t$ if the current belief is $x$ is

$$
f\left(X_{t}^{A}, A_{t}\right)=E\left[\pi(\tilde{\theta}, i) \mid X_{t}^{A}=x, A_{t}=i\right],
$$

where $\pi$ is the expected flow payoff (or utility) if the state of the economy is $\tilde{\theta}$ and the agent has pulled arm $i$. This flow is necessarily linear in $x: f(x, i)=x \pi\left(\theta_{H}, i\right)+(1-x) \pi\left(\theta_{L}, i\right)$.

The assumptions of Theorem 1 are satisfied over any domain $\mathcal{X}_{\varepsilon}=(\varepsilon, 1-\varepsilon)$ with $\varepsilon \in(0,1 / 2)$. Therefore, Theorem 1 implies that $v$ is twice differentiable and solves the HJB equation over any such domain and, therefore, over $(0,1)$.

This shows that the value function of any Brownian multi-armed bandit problem in which the payoff distributions of all arms depend on the same unknown parameter is twice continuously differentiable and solves the HJB equation. This result implies that, as shown in the Appendix (Section $\mathrm{D}$ ), that with finitely many arms, the number of switching points between arms is finite (and bounded above by a function that depends only on the number of arms), and that the optimal control is well defined. It is also used to construct an example with four arms in which a given arm is used on disjoint subsets of the belief domain.

With infinitely many arms, or if the agent can allocate divisible resources across arms, it is a priori unclear whether there exists a well defined optimal control to this problem. Section 4.2 provides a positive answer for a resource allocation problem with two arms.

\section{Existence of an Optimal Control}

The control constructed in Lemma 3 need not be admissible, because it may fail to generate a strong solution to the SDE (1). This section provides conditions for the existence of a maximizing selection that yields an admissible optimal control. All results are based on the following theorem.

THEOREM 2 Suppose that the selection $\hat{a}$ of maximizers is such that the function $\hat{\sigma}: x \mapsto \sigma(x, \hat{a}(x))$ has locally bounded variation. Then, the control $A_{t}^{*}$ that it generates (as defined by Lemma 3), is admissible and optimal.

Proof. Admissibility follows from Nakao (1972), who has shown that a one-dimensional SDE has a unique strong solution if its drift is measurable and its volatility has bounded variation and is 
bounded away from zero (see Footnote 28 for more details on this result). Optimality follows from a standard verification argument.

The bounded variation condition is necessary for the result: Barlow (1982) provides a class of stochastic differential equations for which the volatility does not have bounded variation and there does not exist a strong solution, even if the volatility is bounded below, away from zero 36

An easy case in which $\hat{\sigma}$ has bounded variation is if $\sigma(x, a)$ is independent of $a$, a case that arises in many economic applications, such as the consumption choice problem of Section 3.1 .

Corollary 1 Suppose that $(x, a) \mapsto \sigma(x, a)$ is independent of a. Then, the control $A_{t}^{*}$ generated by the selection $\hat{a}$ of maximizers (as given by Lemma 3) is admissible and optimal.

Proof. The function $x \mapsto \sigma(x)$ is Lipschitz in $x$, by Assumption 3, and has therefore bounded variation. The result then follows from Theorem 2 ,

Beyond Corollary 1, the volatility $\sigma$ may depend on a subset, $\alpha$, of the control. In that case, one way of guaranteeing that $\hat{\sigma}$ has bounded variation is to check that the correspondence $\mathcal{M}$ of maximizers has a selection $\hat{a}=(\hat{\alpha}, \hat{\beta}) \in \mathcal{K}_{1} \times \mathcal{K}_{2} \subset \mathbb{R}^{k_{1}} \times \mathbb{R}^{k_{2}}$, such that $\hat{\alpha}$ has bounded variation 37 and to assume that $\sigma(x, \alpha)$ is uniformly Lipschitz continuous over $\mathcal{X}_{0} \times \mathcal{K}_{1}$ for any compact interval $\mathcal{X}_{0}$ of $\mathcal{X}$. This implies that $\hat{\sigma}$ has bounded variation, as guaranteed by the following result (see Ambrosio and Dal Maso (1990)).

Proposition 3 Suppose that $\sigma$ is uniformly Lipschit 38 continuous on $\mathcal{X}_{0} \times \mathcal{K}_{1}$ for any compact interval $\mathcal{X}_{0}$ of $\mathcal{X}$, and that there exists a selection $\hat{a}(\cdot)=(\hat{\alpha}(\cdot), \hat{\beta}(\cdot))$ of $\mathcal{M}(\cdot)$ such that $\hat{\alpha}$ has bounded variation. Then, $\hat{\sigma}$ has bounded variation.

Guaranteeing that the correspondence $\mathcal{M}$ has a selection with bounded variation would a priori seem a benign and easily satisfied requirement. However, we are not aware of any general result of this kind 39 Similarly, it seems difficult in general to show the existence of an absolutely continuous selection.

\footnotetext{
${ }^{36}$ We are grateful to Nicolai Krylov for pointing this reference out to us.

${ }^{37}$ See Ambrosio and Dal Maso (1990) for the definition of bounded variation for functions defined and taking values in Euclidean spaces. Continuity of the control is neither necessary nor sufficient, as it does not imply bounded variation.

${ }^{38}$ The assumption that $\sigma$ is Lipschitz cannot be easily relaxed: Josephy (1981, Theorem 4) has shown that for the composition $f \circ g$ to have bounded variations for all functions $g$ with bounded variation, $f$ must be Lipschitz.

${ }^{39}$ Chistyakov (2004) defines a concept of bounded variation for correspondences, for which he proves selection theorems guaranteeing a selection of bounded variation. However, we are not aware of any work connecting Chistyakov's concept with the correspondence of maximizers in optimization problems. We are grateful to Vyacheslav Chistyakov for his insights into this problem.
} 
Fortunately, there are more specific strategies to guarantee that the control has bounded variation. If the state space $\mathcal{X}$ can be broken down into finitely many intervals over each of which there is a selection of maximizers such that each component of $\alpha$ is either monotonic or differentiable, this will guarantee the existence of a selection with bounded variation over the entire domain.

THEOREM 3 Suppose that $\sigma$ is uniformly Lipschitz continuous on $\mathcal{X}_{0} \times \mathcal{K}_{1}$, for any compact interval $\mathcal{X}_{0}$ of $\mathcal{X}$, and that $\mathcal{X}$ can be decomposed into disjoint intervals $\left\{\mathcal{X}_{j}\right\}_{j=1}^{n}$ over each of which there is a selection $\hat{a}^{j}=\left(\hat{\alpha}^{j}, \hat{\beta}^{j}\right)$ of $\mathcal{M}$ such that each component of $\hat{\alpha}^{j}$ is either monotonic or differentiable with locally bounded derivative 40 Then, there exists an optimal control, which is Markov and characterized by the selector $\hat{a}$ defined by $\hat{a}(x)=\hat{a}^{j}(x)$ for $x \in \mathcal{X}_{j}$.

Proof. Fixing $j$, let $\left\{\chi_{i}\right\}_{i=0}^{m}$ denote a partition of some compact interval $\mathcal{I}$ of $\mathcal{X}_{j}$ and $\left\{\hat{\alpha}_{k}^{j}\right\}_{k=1}^{k_{1}}$ denote the first $k_{1}$ components of $\hat{a}^{j}$. We have

$$
\sum_{i=0}^{m-1}\left|\hat{\sigma}\left(\chi_{i+1}\right)-\hat{\sigma}\left(\chi_{i}\right)\right| \leq K \sum_{i=0}^{m-1}\left(\left|\chi_{i+1}-\chi_{i}\right|+\sum_{k=1}^{k_{1}}\left|\hat{\alpha}_{k}^{j}\left(\chi_{i+1}\right)-\hat{\alpha}_{k}^{j}\left(\chi_{i}\right)\right|\right)
$$

where $K$ is the Lipschitz constant of $\sigma$ over $\mathcal{I} \times \mathcal{K}_{1}$. Each $\hat{\alpha}_{k}^{j}$ is monotonic or differentiable with locally bounded derivative and, hence, has bounded variation. This and (14) show that $\hat{\sigma}$ has bounded variation over each $\mathcal{X}_{j}$ and, hence, over $\mathcal{X}$. The result then follows from Theorem 2 .

Sections 4.1 and 4.3 provide applications of Theorem 3 in which $\alpha$ is differentiable. Unfortunately, differentiability is violated in many settings, most obviously when the control set $\mathcal{K}$ is discrete. Monotonicity offers an alternative way of exploiting Theorem 3. In many economic problems, the optimal control is monotonic in the state: consumption is increasing in wealth, investment decreases with risk, etc. Establishing such monotonicity without knowing the objective function explicitly is precisely the focus of the theory of monotone comparative statics, as described below.

In what follows, we focus on the case of on the case in which $\mathcal{K}_{1}$ is an interval of $\mathbb{R}$ (i.e., $k_{1}=1$ ). A function $\rho(x, a)$ is supermodular on some domain $\mathcal{X}_{0} \times \mathcal{K}_{1}$ if for all $\tilde{a} \geq a$ in $\mathcal{K}_{1}$ and $\tilde{x} \geq x$ in $\mathcal{X}_{0}$, $\rho(a, x)+\rho(\tilde{a}, \tilde{x}) \geq \rho(\tilde{x}, a)+\rho(x, \tilde{a})$, and submodular if the reverse inequality holds on that domain (or, equivalently, if $-\rho$ is supermodular). When $\rho$ is differentiable in $a$, supermodularity is equivalent to $\rho_{a}$ being nondecreasing in $x$. When $\rho$ is twice differentiable, supermodularity is equivalent to the cross partial being everywhere nonnegative. Supermodularity is a sufficient condition for the maximizer correspondence $x \mapsto \mathcal{M}(x)$ to be nondecreasing in the strong set order, which means that for all $x \leq \tilde{x}, a \in \mathcal{M}(x)$, and $\tilde{a} \in \mathcal{M}(\tilde{x})$, we have $\min \{a, \tilde{a}\} \in \mathcal{M}(x)$ and $\max \{a, \tilde{a}\} \in \mathcal{M}(\tilde{x})$.

\footnotetext{
${ }^{40}$ The derivative is bounded on any compact interval. This condition holds, in particular, if the relevant components of $\hat{\alpha}^{j}$ are continuously differentiable.
} 
In particular, the selections constructed from the smallest and largest maximizers, respectively, are nondecreasing for any supermodular function, and nonincreasing for any submodular one 41

The following result focuses for simplicity on the the case in which $\alpha=a$, so that $\mathcal{K}_{1}=\mathcal{K} \subset \mathbb{R}$.

COROLlary 2 Suppose that $\sigma$ is uniformly Lipschitz continuous on $\mathcal{X}_{0} \times \mathcal{K}$, for any compact interval $\mathcal{X}_{0}$ of $\mathcal{X}$, and let

$$
\rho(x, a)=\frac{1}{\sigma^{2}(x, a)}\left(-r v(x)+f(x, a)+\mu(x, a) v^{\prime}(x)\right),
$$

where $v$ is the value function of the problem, and suppose that $\mathcal{X}$ can be decomposed into $n$ consecutive intervals $\left\{\mathcal{X}_{j}\right\}_{j=1, \cdots, n}$ such that on each product $\mathcal{X}_{j} \times \mathcal{K}, \rho$ is either supermodular or submodular. Then, there exists an optimal control, which is Markov and characterized by a selector $\hat{a}$ of $\mathcal{M}$ such that $\hat{a}$ has bounded variation.

Proof. From (6), maximizing $\rho$ with respect to $a$ is equivalent to maximizing the HJB equation. Let $\hat{a}(x)=\max \mathcal{M}(x)$. Whether $\rho$ is supermodular or submodular on $\mathcal{X}_{j} \times \mathcal{K}, \hat{a}$ is monotonic on this interval. The result follows from Theorem 3 .

REMARK 1 For any strictly positive function $\zeta(x, a)$, note that the HJB equation

$$
0=\max _{a \in \mathcal{K}}-r v(x)+f(x, a)+\mu(x, a) v^{\prime}(x)+\frac{1}{2} \sigma^{2}(x, a) v^{\prime \prime}(x)
$$

has exactly the same maximizers as the equation

$$
0=\max _{a \in \mathcal{K}} \zeta(x, a)\left(-r v(x)+f(x, a)+\mu(x, a) v^{\prime}(x)+\frac{1}{2} \sigma^{2}(x, a) v^{\prime \prime}(x)\right) .
$$

Such transformations are useful for proving monotonicity or differentiability of a selection of maximizers 42

In many economic problems (such as experimentation problems, or when the agent has an increasing concave utility flow), it is possible to show that the value function $v$ has a constant sign, is monotonic, and is either convex or concave 43 This yields the following application of Corollary 2 . Let $l(x, a)=1 / \sigma^{2}(x, a), \bar{f}=l f$, and $\bar{\mu}=l \mu$. Those functions are all primitives of the control problem.

\footnotetext{
${ }^{41}$ This result is easy to check. See Topkis (1978) or Milgrom and Shannon (1994) for a proof. The strong set order is also called the Veinott set order (see Veinott, 1989).

${ }^{42}$ The transformation $\zeta(x, a)=2 / \sigma^{2}(x, a)$ removes $v^{\prime \prime}(x)$ from the maximization problem, and was used for 6 and Corollary 2 Another example, when $\mu$ is known to be strictly positive, is to use $\zeta(x, a)=1 / \mu(x, a)$. This permits to take $v^{\prime}$ out of the maximization problem.

${ }^{43}$ There are many techniques to establish this, either by analyzing the HJB equation, or by constructing various controls to directly show that the value function must be increasing and convex. Applications in this paper provide several such examples.
} 
Corollary 3 Suppose that i) $\sigma$ is uniformly Lipschitz continuous on $\mathcal{X}_{0} \times \mathcal{K}$, for any compact interval $\mathcal{X}_{0}$ of $\mathcal{X}$, ii) $v$ is nonnegative, increasing, and convex, and iii) $\bar{\mu}$ is nondecreasing in a and supermodular, $\bar{f}$ is supermodular, $l$ nonincreasing in a and submodular. Then, there exists an optimal control, and this control is Markov and nondecreasing in $x$.

The corollary is straightforward to prove: its conditions guarantee that each term in $(15)$ is supermodular.

The supermodularity assumed in Corollary 2 can be weakened in several ways. Indeed, it suffices that $\rho$ satisfies the single-crossing property or Interval Dominance Order (IDO) property in $(a, x)$ for asserting the existence of a monotonic selection on any given interval 44 Here is a useful way of checking these properties: when $\rho$ is differentiable with respect to $a$, the IDO property is guaranteed to hold ${ }^{45}$ over $\mathcal{K} \times \mathcal{I}$, where $\mathcal{I}$ is any interval of $\mathcal{X}$, if there exists a positive, nondecreasing function $\gamma_{\mathcal{I}}(\cdot)$ of $a$ such that, for each $x^{\prime \prime}>x^{\prime}, \rho_{a}\left(x^{\prime \prime}, a\right) \geq \gamma_{\mathcal{I}}(a) \rho_{a}\left(x^{\prime}, a\right)$. If $\gamma_{\mathcal{I}}$ is constant over $\mathcal{K}$, the inequality implies that $\rho$ satisfies the single crossing property in $(a, x)$. If $\gamma_{\mathcal{I}}$ is identically equal to 1 , we recover the supermodularity condition.

\subsection{Example: Optimal Growth with Consumption and Portfolio Optimization}

We reconsider the example of Section 3.1. The agent chooses, in addition to consumption, the fraction $\varphi_{t} \in[0,1]$ of his wealth going to a risky asset, so that $A_{t}=\left(C_{t}, \varphi_{t}\right){ }^{46}$ The volatility $\sigma\left(X_{t}, A_{t}\right)$ is now increasing in $\varphi_{t}$, and we cannot apply Corollary 1 anymore to prove the existence of an optimal control. However, if one can show that the agent's risk-taking behavior is nondecreasing in his capital holdings, this will imply monotonicity of $\varphi$, and guarantee the existence of an optimal control, by Theorem 3 . To guarantee nonnegativity of the capital process $X$, we assume that that the agent cannot consume when $x=0$. This does not affect Theorem 1, as long as the feasible consumption set $[0, \bar{c}(x)]$ is continuous in $x{ }^{47}$

\footnotetext{
${ }^{44}$ The single-crossing property (Milgrom and Shannon, 1994), generalizes supermodularity (Topkis, 1978), and is itself generalized by the IDO property (Quah and Strulovici, 2009). Comparative statics also obtain for objective functions that are quasi-concave and ordered by the location of their peaks, as studied by Karlin and Rubin (1956) and Lehmann (1988). This latter ordering is also generalized by the IDO property.

${ }^{45}$ See Quah and Strulovici (2009, Proposition 2).

${ }^{46}$ We are ruling out short sales and borrowing, which guarantees that $\varphi_{t}$ lies in the compact space $[0,1]$.

${ }^{47}$ See Footnotes 16 and 26 . We can, for example, take $\bar{c}(x)$ to be any continuous function that rapidly increases from $\bar{c}(0)=0$ to some positive constant $\bar{c}$. For the concavity argument below (Footnote 52 , we also require that $\bar{c}(\cdot)$ be concave.
} 
Slightly simplifying the setting, the HJB equation for this problem is

$$
r v(x)=\max _{(c, \varphi) \in[0, \bar{c}(x)] \times[0,1]}\left\{u(c)+[\mu(x, \varphi)-c] v^{\prime}(x)+\frac{1}{2} \sigma^{2}(x, \varphi) v^{\prime \prime}(x)\right\} .
$$

Thus, the optimal $\varphi$ maximizes the objective

$$
\mu(x, \varphi) v^{\prime}(x)+\frac{1}{2} \sigma^{2}(x, \varphi) v^{\prime \prime}(x) .
$$

If this objective function satisfies the single crossing property in $(\varphi, x)$, there must exist a nondecreasing maximizing selection $\varphi$. If $\sigma$ is Lipschitz continuous in $\varphi$, Theorem 3 then implies that there exists an optimal control.

The single crossing property holds if i) $v$ is increasing and concave 48 ii) $\mu$ and $\sigma$ are increasing in $\varphi$, and iii) for any $\varphi_{1}<\varphi_{2}$

$$
\frac{-v^{\prime \prime}(x) \Delta \sigma^{2}(x)}{v^{\prime}(x) \Delta \mu(x)}
$$

is decreasing in $x$, where $\Delta \sigma^{2}(x)=\sigma^{2}\left(x, \varphi_{2}\right)-\sigma^{2}\left(x, \varphi_{1}\right)$ and $\Delta \mu(x)=\mu\left(x, \varphi_{2}\right)-\mu\left(x, \varphi_{1}\right)$. Single crossing is then a direct consequence of Proposition 1 in Quah and Strulovici (2012a) ${ }^{49}$ In turn, (16) holds if $-v^{\prime \prime}(x) / v^{\prime}(x)$ and $\Delta \sigma^{2}(x) / \Delta \mu(x)$ are both decreasing. The first condition means that the agent has decreasing absolute risk aversion in $x$, while the second condition is immediate to check for any given drift and volatility functions $\mu$ and $\sigma 50$ Thus, we recover the intuition, stated above, that if the agent's risk-aversion is decreasing in his capital, the control $\varphi$ is monotonic and there exists an optimal control.

The previous result relied on the value function exhibiting decreasing absolute risk aversion, which is an endogenous property. The next approach avoids this problem. It proves differentiability of $\varphi$, exploiting another possibility offered by Theorem 3 .

We specialize the model to a version of Merton's consumption and investment problem 51 The safe asset has a constant return $\mu_{0}$, while the a risky asset has a payoff that follows a geometric Brownian motion with a higher return $\mu>\mu_{0}$. We impose the condition $\mu<r$, which is standard in the portfolio optimization literature and is equivalent to the inequality $K_{2}^{\mu}<r$ of Assumption 3 . The agent's capital follows the equation

$$
d X_{t}=\left[X_{t}\left(\left(\mu-\mu_{0}\right) \varphi_{t}+\mu_{0}\right)-C_{t}\right] d t+X_{t} \varphi_{t} \sigma d B_{t}
$$

\footnotetext{
${ }^{48}$ Such properties may be shown from direct arguments, as in Footnote 52

${ }^{49}$ Conditions i) and ii) guarantee that the functions $v^{\prime \prime}(x) \Delta \sigma^{2}(x)$ and $v^{\prime}(x) \Delta \mu(x)$ each have a constant sign and, thus, are single crossing functions. Condition iii) is the signed-ratio monotonicity condition in Quah and Strulovici (2012a), which guarantees that the sum is also a single crossing function.

${ }^{50}$ That condition can be interpreted as a sort of increasing marginal Sharpe ratio: the additional exposure to risk from moving from $\varphi_{1}$ to $\varphi_{2}$, has a better Sharpe ratio for higher capital levels than lower ones.

${ }^{51}$ See Merton $(1969,1971)$ and Duffie $(2001)$ for a general presentation.
} 
The agent has a twice differentiable flow utility $u(\cdot)$ such that $u^{\prime}(c)>0$ and $u^{\prime \prime}(c)<0$ for all $c \geq 0$. The HJB equation is

$$
r v(x)=\max _{(c, \varphi) \in[0, \bar{c}(x)] \times[0,1]} u(c)+\left(x\left(\mu-\mu_{0}\right) \varphi+x \mu_{0}-c\right) v^{\prime}(x)+\frac{1}{2} x^{2} \sigma^{2} \varphi^{2} v^{\prime \prime}(x) .
$$

It is easily shown that $v^{\prime}(x)>0$. The optimal consumption $c(x)$ therefore satisfies the first-order condition

$$
u^{\prime}(c)=v^{\prime}(x)
$$

or equals the corner value $\bar{c}(x)$. It is also easily shown that the value function is concave ${ }^{52}$ There is a unique maximizer $\varphi(x)$ to the HJB equation, given by 53

$$
\varphi(x)=\min \left\{-\frac{v^{\prime}(x)}{x v^{\prime \prime}(x)} \frac{\mu-\mu_{0}}{\sigma^{2}}, 1\right\} .
$$

Proposition 4 The maximizing selection $(c(x), \varphi(x))$ of the HJB equation generates an admissible, optimal control.

Proof. It suffices to show that $\varphi$ has bounded variation on $\mathcal{I}_{\varepsilon}=\{x \in(\varepsilon, 1 / \varepsilon): \varphi(x) \in(0,1)\}$, which consists of disjoint open intervals, for each $\varepsilon<1$. Following Theorem 3 , it suffices to show that $\varphi$ is continuously differentiable on that domain. Rewriting the HJB equation, we hav $\varrho^{54}$

$$
v^{\prime \prime}(x)=-\max _{(c, \varphi) \in[0, \bar{c}(x)] \times[0,1]}\left\{\frac{2}{x^{2} \sigma^{2} \varphi^{2}}\left(u(c)+\left(x\left(\mu-\mu_{0}\right) \varphi+x \mu_{0}-c\right) v^{\prime}(x)-r v(x)\right)\right\} .
$$

Since the maximizers $c(x)$ and $\varphi(x)$ are unique, Corollary 4, Part iii) of Milgrom and Segal (2002) implies that $v^{\prime \prime}$ is differentiable at all $x>0$. Moreover, the derivative is continuous, as is easily checked. Therefore, (18) implies that $\varphi$ is continuously differentiable on $\mathcal{I}_{\varepsilon}$.

\subsection{Example: Multi-Armed Bandit and Resource Allocation}

We now reconsider the bandit problem of Section 3.2 with two arms, but in which the agent has a fixed resource, normalized to 1 , to allocate between the arms at each time 55 As before, each

\footnotetext{
${ }^{52}$ Concavity is established as follows: for $0<x^{1}<x^{2}$ and $\lambda \in(0,1)$, let $x=\lambda x^{1}+(1-\lambda) x^{2}$. Consider any admissible controls $\varphi^{1}, C^{1}$ and $\varphi^{2}, C^{2}$ chosen starting from $x^{1}$ and $x^{2}$, respectively. Let $\varphi_{t}=\left(\lambda X_{t}^{1} \varphi_{t}^{1}+(1-\right.$ $\left.\lambda) X_{t}^{2} \varphi_{t}^{2}\right) /\left(\lambda X_{t}^{1}+(1-\lambda) X_{t}^{2}\right) \in[0,1]$ and $C_{t}=\lambda C_{t}^{1}+(1-\lambda) C_{t}^{2}$. It is easy to check that the admissible control $(\varphi, C)$ starting from $x$ yields $X_{t}=\lambda X_{t}^{1}+(1-\lambda) X_{t}^{2} \geq 0$ for all $t$. Moreover, $u\left(C_{t}\right) \geq \lambda u\left(C_{t}^{1}\right)+(1-\lambda) u\left(C_{t}^{2}\right)$, by concavity of $u$. Discounting and integrating proves the claim.

${ }^{53}$ If $v^{\prime \prime}(x)=0$, the first term of the minimization is assumed to be equal to $+\infty$.

${ }^{54}$ The optimal allocation $\varphi(x)$ is strictly positive for all $x$, since $v^{\prime}(x)>0$.

${ }^{55}$ Examples of experimentation with resource allocations include Bolton and Harris (1999) and Keller et al. (2005). In their setting, one arm is safe, whereas both arms are "risky" in the present model.
} 
arm has a payoff distribution that depends on the common parameter $\tilde{\theta}$, whose belief at time $t$ is the state $X_{t}=\operatorname{Pr}\left(\tilde{\theta}=\theta_{H} \mid \mathcal{F}_{t}\right)$. The control of the agent is now a fraction $A_{t} \in[0,1]$ allocated by the agent to the first arm. Notice that each allocation $a \in[0,1]$ yields a combination of two signals which may be aggregated into a single signal with volatility $\sigma(x, a)=\xi(a) x(1-x)$ about the parameter $\tilde{\theta}$, and yields an expected payoff $f(x, a)$. Thus, we are exactly in the setting of Section 3.2 , with $\mathcal{K}=[0,1]$. In particular, the value function of the problem is twice continuously differentiable and solves everywhere on $(0,1)$ the HJB equation

$$
0=\max _{a \in[0,1]} x f_{H}(a)+(1-x) f_{L}(a)+\frac{1}{2}(x(1-x))^{2} \xi^{2}(a) v^{\prime \prime}(x)-r v(x) .
$$

This equation may be rewritten as

$$
v^{\prime \prime}(x)=\frac{2}{(x(1-x))^{2}} \max _{a \in[0,1]}\left\{\frac{1}{\xi^{2}(a)}\left(x f_{H}(a)+(1-x) f_{L}(a)-r v(x)\right)\right\} .
$$

Therefore, the existence of a monotone optimal selection will be guaranteed if the function

$$
\frac{-r v(x)}{\xi^{2}(a)}+\frac{x f_{H}(a)+(1-x) f_{L}(a)}{\xi^{2}(a)}
$$

is either supermodular or submodular in $(a, x)$.

Assuming that $\Delta f(a)=f_{H}(a)-f_{L}(a) \geq 0$ for all $a$ (i.e., $\theta_{H}$ is the "good" state of the economy), $v$ is nondecreasing in $x$. Therefore, the first term in (21) is supermodular (submodular) if and only if $\xi(a)$ is increasing (decreasing) in $a$. Similarly, the second term is supermodular (submodular) in $(x, a)$ if $\Delta f(a) / \xi^{2}(a)$ is increasing (decreasing) in $a$. Combining these observations with Theorem 3 proves the following result.

Proposition 5 Suppose that $\xi(a)$ and $\Delta f(a) / \xi^{2}(a)$ are either both increasing or both decreasing in a. Then there exists a monotone optimal selection $a(x)$ and, therefore, an optimal control.

\subsection{Existence of an Optimal Contract in Principal Agent Problems}

This application revisits the seminal analysis contained in Sannikov (2008). The objective here is to provide conditions under which the optimal contract characterized in that paper generates a strong solution for the continuation value process of the agent. The existence problem discussed here is actually relevant for many recent continuous-time principal-agent models. In the recursive approach to principal-agent models, the principal is viewed as "controlling" the continuation value of the agent by providing him with consumption, and rewarding or punishing him depending on his output. To save space, we only sketch the presentation and arguments already contained in Sannikov (2008), and refer the reader to that paper for a detailed exposition. 
The principal chooses a consumption process $\left\{C_{t}\right\}_{t \geq 0}$ and can implement, by choosing the right incentives, some effort process $\left\{A_{t}\right\}_{t \geq 0}$. Precisely, assuming that the agent's continuation utility $W_{t}$ is adapted to the filtration generated by the exogenous uncertainty, the Martingale Representation Theorem implies that

$$
d W_{t}=\left(r W_{t}-u\left(C_{t}\right)+h\left(A_{t}\right)\right) d t+\psi\left(A_{t}\right) d B_{t}
$$

where $A_{t}$ is the level of effort that the principal chooses to implement, $u$ and $h$ are the utility and effort cost functions of the agent, and $\psi(a)=h^{\prime}(a)$ is the contract sensitivity to observed output. The principal seeks to maximize the payoff

$$
F_{0}=E\left[\int_{0}^{\tau} e^{-r t}\left(A_{t}-C_{t}\right) d t\right]
$$

subject to 22 and some initial participation constraint $W_{0}=w$.

Therefore, the principal faces an optimal control problem in which the state is $W_{t}$ and the controls are $C_{t}$ and $A_{t}$, following the standard notation in principal-agent models 56 Sannikov has shown that the agent is "retired" when $W_{t}$ hits some upper bound $\bar{w}$. The optimal consumption $c(w)$ level is easily shown to be bounded by some level $\bar{c}$ on $[0, \bar{w}]$, while the effort domain is assumed to be bounded above by some level $\bar{a}$.

The principal's HJB equation on $(0, \bar{w})$ is

$$
r F(w)=\max _{(a, c) \in[0, \bar{a}] \times[0, \bar{c}]}\left\{a-c+F^{\prime}(w)(r w-u(c)+h(a))+\frac{1}{2} \psi(a)^{2} F^{\prime \prime}(w)\right\} .
$$

Under standard assumptions on $u$ and $h$, Sannikov shows that the HJB equation has a solution $F$ that is twice continuously differentiable. Moreover, $F$ is strictly concave.

At the optimal contract, standard results imply that there exists a weak solution to the SDE (22), whenever $\psi(a(w))$ is bounded away from zero 57 With a weak solution, the continuation value process is not necessarily adapted to the filtration generated by the Brownian Motion $B_{t}$, which implies that there is more randomness in $W_{t}$, and therefore the contract, than generated by the output process.

One possible interpretation for having (only) a weak solution is that the principal is randomizing over continuation utility levels. However, the principal's objective $F(w)$ is strictly concave, which implies that he is risk-averse along the optimal contract. This makes this interpretation problematic as a justification for a weak solution. The uncertainty arising from the broader filtration implied by

\footnotetext{
${ }^{56}$ Thus, what has been called " $x$ " in earlier section is now " $w$ ", whereas " $A$ " now refers to only one component of the bidimensional control. $F$, instead of $v$ denotes the value function of the problem.

${ }^{57}$ See, e.g., Revuz and Yor (2001, Corollary 1.12, p. 372).
} 
weak solutions seems conceptually different from the one obtained if the principal explicitly allows all contractual variables to depend on other sources of uncertainty, such as a second Brownian motion.

Another issue is that, as observed by Sannikov (2008, Footnote 29), the application of the Martingale Representation Theorem, which is a key step in the analysis, must also be modified to include the larger filtration. In that case, the resulting representation of $W$ cannot a priori be reduced to a stochastic integral with respect to the initial Brownian motion. As a result, the analysis of the contract, which was largely based on choosing the integrand of that stochastic integral, must be modified. Intuitively, it raises the issue of what "sensitivity" the principal should apply to the new sources of uncertainty. Guaranteeing a strong solution gets rid of all these issues.

Proposition 6, below, provides simple conditions on the effort cost function $h(a)$ under which the continuation value process has a unique strong solution. Thus, $W_{t}$ is adapted to $\mathcal{F}_{t}^{B}$, and the contract does not involve randomization.

Proposition 6 Suppose that the effort cost function $h$ is increasing, three times differentiable, and satisfies $h(0)=0, h^{\prime}(0)>0, h^{\prime \prime}(a)>0$ and $h^{\prime \prime \prime}(a) \geq 0$ for all $a \in(0, \bar{a}]$. Then, the $S D E$

$$
d W_{t}=\left(r W_{t}-u\left(C_{t}\right)+h\left(A_{t}\right)\right) d t+\psi\left(A_{t}\right) d B_{t}
$$

has a unique strong solution.

The assumptions on $h$ are satisfied, for example, if $h(a)=\gamma_{1} a^{p}+\gamma_{0} a$ for $p \geq 2$ and $\gamma_{0}, \gamma_{1}>0$, as in Sannikov's (2008) numerical example, if it is exponential, or is equal to any positive combination of such functions.

Proof. Because consumption appears only in the drift of 22, it suffices to show that $a(w)$ has bounded variation. The optimality equation for $a$ can be reduced to the optimization problem

$$
\max _{a \in[0, \bar{a}]} a+F^{\prime}(w) h(a)+F^{\prime \prime}(w) \frac{1}{2} \psi(a)^{2} .
$$

We will first show that the objective function in (24) is strictly quasiconcave in $a$, for each $w$. That is, its derivative with respect to $a, \Psi(a, w)=1+F^{\prime}(w) h^{\prime}(a)+F^{\prime \prime}(w) \psi^{\prime}(a) \psi(a)$, can cross 0 at most once as $a$ increases, from above. We have, recalling that $\psi(a)=h^{\prime}(a)$,

$$
\Psi_{a}(a, w)=F^{\prime}(w) h^{\prime \prime}(a)+F^{\prime \prime}(w)\left(h^{\prime \prime \prime}(a) h^{\prime}(a)+h^{\prime \prime}(a)^{2}\right)
$$

Our assumptions imply that $h^{\prime}(a)>0$ for all $a$. We can rewrite the previous equation as

$$
\Psi_{a}(a, w)=\frac{\Psi(a, w)-1}{h^{\prime}(a)} h^{\prime \prime}(a)+F^{\prime \prime}(w) h^{\prime \prime \prime}(a) h^{\prime}(a) .
$$


Whenever $\Psi(a, w) \leq 0$, the previous equation implies that

$$
\Psi_{a}(a, w) \leq-\frac{h^{\prime \prime}(a)}{h^{\prime}(a)}+F^{\prime \prime}(w) h^{\prime \prime \prime}(a) h^{\prime}(a) .
$$

Since $h^{\prime \prime}(a)>0$ and $h^{\prime \prime \prime}(a) \geq 0$ for $a>0$, and $F$ is concave, we conclude that $\Psi_{a}(a, w)<0$ whenever $\Psi(a, w) \leq 0$ and $a>0$, proving strict quasiconcavity of the objective function in (24).

This also shows uniqueness of the optimizer $a(w)$. Moreover, $a(w)$ is continuous in $w$, from (24) and Berge's maximum theorem, whose conditions are easily checked here. Therefore, it suffices to show that $a(w)$ has bounded variation on any interval $\left(w_{1}, w_{2}\right)$ over which $a(w) \in(0, \bar{a})$. By Theorem 3, we will have proved the result if we show that $a(w)$ is continuously differentiable on any such interval.

The optimal effort is determined on $\left(w_{1}, w_{2}\right)$ by the first-order condition $\Psi(a(w), w)=0$. To show continuous differentiability of $a(w)$, we first observe that the optimal consumption $c(w)$ is also unique, as an immediate consequence of $(23)$ and the fact that $u^{\prime}(c)$ is strictly decreasing in $c$. An envelope theorem of Milgrom and Segal (2002, Corollary 4, Part iii)) then implies that $F$ is three times continuously differentiable and, hence, that $\Psi$ is differentiable with respect to $w \sqrt[58]{\text { Because }}$ $\Psi_{a}(a, w)$ is strictly negative, evaluated at $a(w)$, the implicit function theorem can be applied to the first-order condition, showing that $a$ is continuously differentiable in $w$ on the desired domain.

\section{Optimal Stopping and Smooth Pasting}

This section establishes, under conditions similar to those of Section 2 , that the value function of any optimal stopping problem is continuously differentiable. In particular, it is differentiable at any threshold at which stopping becomes optimal, which is the smooth pasting property. For clarity, we separate optimal control and optimal stopping problems. The problems can be combined with an appropriate extension of Theorems 1 and $4 t^{59}$

Consider the optimal stopping problem

$$
v(x)=\sup _{\tau \in \mathcal{T}} E\left[\int_{0}^{\tau} e^{-r t} f\left(X_{t}\right) d t+e^{-r \tau} g\left(X_{\tau}\right)\right],
$$

\footnotetext{
${ }^{58}$ The envelope theorem is applied to the modified HJB equation $F^{\prime \prime}(w)=-\max _{a, c}\left\{2 / \psi(a)^{2}\left[a-c+F^{\prime}(w)(r w-\right.\right.$ $u(c)+h(a))-r F(w)]\}$. Our assumptions on $h$ guarantee that $\psi(a)=h^{\prime}(a)$ is bounded below by $h^{\prime}(0)>0$ and, hence, that the previous equation holds on $\left(w_{1}, w_{2}\right)$.

${ }^{59}$ In the combined problem, the optimal continuation region consists of disjoint open intervals, as in this section, and on any such interval, the analysis of Section 2 can be applied, showing that value function of the controlled process is twice differentiable. The analysis of the present section can then be replicated to show that the value function is everywhere $C^{1}$, replacing the dynamic equation 25 by the HJB equation, which is satisfied on the optimal continuation region.
} 
where $\mathcal{T}$ is the set of all stopping times adapted to the initial filtration $\mathcal{F}$, and $\left\{X_{t}\right\}_{t \in \mathbb{R}_{+}}$solves the equation

$$
d X_{t}=\mu\left(X_{t}\right) d t+\sigma\left(X_{t}\right) d B_{t}
$$

subject to the initial condition $X_{0}=x$. We maintain the same assumptions as before on $\mu, \sigma$ and $f$, which guarantee that the SDE has a unique strong solution, and, along with Assumption 4 , that the expected payoff is well defined for all stopping times.

Assumption $4 g$ is $C^{1}$ and $|g(x)| \leq K^{g}(1+|x|)$ for some constant $K^{g} \geq 0$.

TheOREM 4 Under Assumptions 2- 4: 60 is continuously differentiable on the interior of $\mathcal{X}$.

Proof. Let $\mathcal{Y}$ denote the subset of $\mathcal{X}$ for which $v(x)=g(x)$. $\mathcal{Y}$ consists of all the states at which it is optimal to stop immediately. By continuity of $v$ and $g, \mathcal{X} \backslash \mathcal{Y}$ consists of disjoint open intervals $\left\{\mathcal{Z}_{i}\right\}_{i \in I}$. Pick any two points $x_{1}<x_{2}$ in such an interval. The result stated in Appendix B guarantees the existence of a $C^{2}$ solution $w$ to the ordinary differential equation

$$
w^{\prime \prime}(x)+\frac{1}{\frac{1}{2} \sigma(x)^{2}}\left(-r w(x)+f(x)+\mu(x) w^{\prime}(x)\right)=0
$$

with boundary conditions $w\left(x_{1}\right)=v\left(x_{1}\right)$ and $w\left(x_{2}\right)=v\left(x_{2}\right)$. A standard verification argument then shows that $v$ coincides with $w$ on any such interval and, therefore, that $v$ is $C^{2}$ on such interval and, hence, on $\mathcal{X} \backslash \mathcal{Y}=\cup_{i \in I} \mathcal{Z}_{i}$.

Consider now the boundary of some interval $\mathcal{Z}_{i}$, for example the upper boundary, and call it $x^{*}$. By construction, $v(x)>g(x)$ for $x$ in a left-neighborhood of $x^{*}$, and $v\left(x^{*}\right)=g\left(x^{*}\right)$. In particular, $v_{l}^{\prime}\left(x^{*}\right)$, the left derivative of $v$ at $x^{*}$, must be less than or equal to $g^{\prime}\left(x^{*}\right)$.

To show that the inequality is tight, suppose, by contradiction, that $v_{l}^{\prime}\left(x^{*}\right)<g^{\prime}\left(x^{*}\right)$, and consider the domain $\left[x_{1}, x_{2}^{*}=x^{*}+\varepsilon\right]$ for some $x_{1}$ in $\mathcal{Z}_{i}$ and some small $\varepsilon>0$. From Appendix $\mathrm{B}$, there exists a solution $w$ to Equation 25 on $\left[x_{1}, x_{2}^{*}\right]$, with initial value $w\left(x_{1}\right)=v\left(x_{1}\right)$ and initial slope $w^{\prime}\left(x_{1}\right)=v^{\prime}\left(x_{1}\right)$. Moreover, this solution satisfies $w\left(x^{*}\right)=v\left(x^{*}\right)$, and $w^{\prime}\left(x^{*}\right)=v_{l}^{\prime}\left(x^{*}\right)$, because $v$ solves the same initial value problem (IVP) on the domain $\left[x_{1}, x^{*}\right]$, and the solution is unique (see Lemma 4). Therefore, $w(x)<g(x)$ for $x$ in a right neighborhood of $x^{*}$, and without loss, on $\left(x^{*}, x_{2}^{*}\right)$. Taking a slightly higher slope $\hat{s}>s^{*}$, consider the solution $\hat{w}$ to the IVP on the domain $\left[x_{1}, x_{2}^{*}\right]$ with initial slope $\hat{s}$ and initial value $v\left(x_{1}\right)$. For $\hat{s}$ close to $s$, this solution hits $g$ at some $\hat{x} \in\left(x_{1}, x_{2}^{*}\right)$, because solutions to the IVP are continuous in $s$ (see Lemma 5 in the Appendix). Moreover Lemma 9, also proved in the Appendix, implies that $\hat{w}(x)>w(x)$ for all $x \in\left(x_{1}, x^{*}\right]$ and, therefore, that $\hat{x}>x^{*}$. We redefine $\hat{w}$ by setting $\hat{w}(x)=g(x)$ for all $x>\hat{x}$. By construction,

\footnotetext{
${ }^{60}$ The pure stopping problem that we consider is equivalent to reducing the control set $\mathcal{K}$ of Section 2 to a singleton.
} 
$\hat{w}\left(x^{*}\right)>g\left(x^{*}\right)=v\left(x^{*}\right)$. Moreover, $\hat{w}$ corresponds to the expected payoff obtained if the following stopping strategy is used: starting from $x^{*}$, continue until either $x_{1}$ or $\hat{x}$ is reached. If $\hat{x}$ is reached first, stop. If $x_{1}$ is reached first, follow the initial strategy leading to value $v\left(x_{1}\right)$. This strategy thus gives, starting from $x^{*}$, a strictly higher expected payoff than $v\left(x^{*}\right)$, yielding a contradiction. This shows that $v$ is differentiable at $x$ in the following cases: i) $x$ lies in the interior of some interval $\mathcal{Z}_{i}$, ii) $x$ connects two intervals $\mathcal{Z}_{i}$ and $\mathcal{Z}_{j}$ (i.e., it is the upper bound of one interval, and the lower bound of the other), and iii) $x$ is a bound of some interval $\mathcal{Z}_{i}$, and $v(y)=g(y)$ in some neighborhood on the other side of $x$. Moreover, in cases ii) and iii) the derivative is given by $v^{\prime}(x)=g^{\prime}(x)$. In all these cases, the derivative is continuous at $x$ because the solution to 25 on any $\mathcal{Z}_{i}$ is twice differentiable and because $g$ is $C^{1}$.

There remains to show the result when $x$ is an accumulation point of stopping and continuation regions. This is proved in Section C.1 of the Appendix.

\subsection{Example: Option Value}

A simple application of the smooth pasting property is to prove, under great generality, the wellknown principle that when the value function of any stopping problem is convex, the agent waits beyond the myopic optimum to stop, due to an "option value of waiting." This principle arises in investment decisions (Dixit, 1989)), experimentation problems ${ }^{61}$ the exercise of American and real options, and bankruptcy decisions (Leland, 1994).

Formally, suppose that the terminal value function is a constant: $g(x)=\bar{g}$ for all $x$, and suppose that it can be established by some means, that the value function $v$ of the agent is convex 62 Consider any maximal interval $\mathcal{Z}$ over which continuing is strictly optimal, as in the proof of Theorem 4 , with endpoints $\underline{x}$ and $\bar{x}{ }^{63}$ Theorem 4 implies that for any finite endpoint of $\mathcal{Z}$, say $\bar{x}$, the smooth pasting condition holds, i.e., $v^{\prime}(\bar{x})=g^{\prime}(\bar{x})=0$. Moreover, the Bellman equation on $\mathcal{Z}$ implies that

$$
0=-r v(\bar{x})+f(\bar{x})+\mu(x) v^{\prime}(\bar{x})+\frac{1}{2} \sigma^{2}(x) v_{l}^{\prime \prime}(\bar{x}),
$$

where $v_{l}^{\prime \prime}(\bar{x})$ is the left second derivative of $v$ at $\bar{x}$, which was shown earlier to always exist under

\footnotetext{
${ }^{61}$ In experimentation problems with a risky and a safe arm, moving to the safe arm amounts to a stopping problem. See e.g., Bolton and Harris (1999).

${ }^{62}$ Typical arguments include direct reasoning on the strategy of the agents, or reasoning based on the dynamic equation for $v$. One version of the former approach is similar to the argument given in Footnote 52 , but reversing it: pick any stopping policy that is optimal for $x$, and show that the convex combination of the value starting from $x_{1}<x_{2}$, following $x$, dominates $v(x)$. This will work if the process $X_{t}$ is linear in the initial condition $x$ (as is the case for geometric Brownian motion), and if the flow and terminal payoffs are convex in their argument.

${ }^{63}$ As shown in that proof, the strict continuation domain always consists of disjoint open intervals.
} 
the assumptions of Theorem 4. Convexity of $v$ and smooth pasting then imply that

$$
f(\bar{x}) \leq r \bar{g},
$$

with a strict inequality if $v_{l}^{\prime \prime}(\bar{x})>0$. This shows that the option value of waiting is positive. To see this, suppose first that $\bar{g}=0$ (stopping yields a zero lump sum). From (26), the agent stops when his current flow payoff is negative. If $\bar{g}$ is nonzero, the same idea applies, where $r \bar{g}$ is the flow-payoff equivalent of stopping. The result holds for any shape of the stopping region.

\section{Smoothness, Envelope Theorem, and Dynamic Sensitivity Equa- tion for Parameterized Stopping Problems}

In analyzing economic models, a frequent objective is to describe how the value function of some optimization problem changes with parameters of the problem, which is often based on an application of the Envelope Theorem. This section provides such an envelope theorem and a dynamic "sensitivity" equation for the value function of optimal stopping problems.

We consider the following parameterized optimal stopping problem in which the parameter $\theta$ lies an open subset $\Theta$ of $\mathbb{R}$ :

$$
v(x, \theta)=\sup _{\tau \in \mathcal{T}} E\left(\int_{0}^{\tau} e^{-r t} f\left(X_{t}, \theta\right) d t+e^{-r \tau} \phi\left(X_{t}, \theta\right)\right)
$$

subject to $d X_{t}=\mu\left(X_{t}, \theta\right) d t+\sigma\left(X_{t}, \theta\right) d B_{t}$ and $X_{t} \in[0,+\infty)$.

We also assume that 0 is an absorbing state, and that the payoff and termination values at $x=0$ are equal to 0 for all $\theta$.

Extending the setting of Section 5 , we assume that $f(\cdot, \theta), g(\cdot, \theta), \mu(\cdot, \theta)$, and $\sigma(\cdot, \theta)$ satisfy the assumptions of Theorem 4, uniformly in $\theta$, and that $g(\cdot, \theta)$ is twice continuously differentiable, for each $\theta$. We also assume that, for each $x, f(x, \cdot), g(x, \cdot), \mu(x, \cdot)$, and $\sigma(x, \cdot)$ are differentiable in $\theta$, and that the derivatives in $\theta$ are continuous in $(x, \theta)$.

Our main assumption is a strict single crossing condition, which guarantees that continuing is strictly optimal for low states and strictly suboptimal for high states ${ }^{64}$ Let

$$
h(x, \theta)=r g(x, \theta)-f(x, \theta)-\mu(x, \theta) g^{\prime}(x, \theta)-\frac{1}{2} \sigma^{2}(x, \theta) g^{\prime \prime}(x, \theta) .
$$

\footnotetext{
${ }^{64}$ Pham (2009) and Villeneuve (2007) use similar conditions (with and without flow payoffs, respectively) to show that the stopping region is a half line. Here, the strict single crossing condition is used to prove a slightly stronger result: it is strictly suboptimal to continue beyond the lower bound of the stopping region. This uniqueness is needed to prove differentiability of the value function with respect to the parameter.
} 
Assumption 5 For each $\theta$, there exists $\underline{x}(\theta)$ such that $h(x, \theta)<(>) 0$ for $x<(>) \underline{x}(\theta)$.

Assumption 5 is a condition on the primitives of the stopping problem, and can be easily checked. For example, it holds if $g$ is linear and strictly increasing in $x$, and $f$ and $\mu$ are weakly decreasing in $x$. More generally, techniques guaranteeing that $h$ has the single crossing condition can be found in the comparative statics literature 65

The key result implied by Assumption 5 is the uniqueness of an optimal stopping threshold.

Since some arguments below rely on compactness of the domain, we also assume that there exists a uniform upper bound $\bar{x}$ such that continuing above $\bar{x}$ is strictly suboptimal for all $\theta$. This assumption holds if there exists $\hat{x}$ and $A>0$ such that $\inf _{[\hat{x},+\infty)} r g(x) \geq \sup _{[\hat{x},+\infty)} f(x)+A 66$

Proposition 7 Under Assumption 5, there exists, for each $\theta$, a threshold $x(\theta)$ such that continuing is strictly optimal for $x<x(\theta)$ and continuing for a strictly positive amount of time is strictly suboptimal for $x>x(\theta)$. Moreover, $x(\theta) \geq \underline{x}(\theta)$ for all $\theta$ and $x(\theta)$ is continuous in $\theta$.

Proof. Pham (2009, Lemma 5.2.6) proves, under a slightly weaker condition than Assumption 5. that the set of $x$ 's for which stopping is weakly optimal has the form $[x(\theta),+\infty)$ for some $x(\theta) \geq$ $x(\theta) 67$ Suppose, by contradiction, that there is some $x>x(\theta)$ starting from which continuing for a strictly positive amount of time is also optimal. This implies that there exists an open interval, $\mathcal{J}$, containing $x$, over which both continuing and stopping are optimal. Since continuing is optimal, the value function $v(\cdot, \theta)$ solves the equation

$$
r v(x, \theta)=f(x, \theta)+\mu(x, \theta) v^{\prime}(x, \theta)+\frac{1}{2} \sigma^{2}(x, \theta) v^{\prime \prime}(x, \theta)
$$

over $\mathcal{J}$. Since stopping is also optimal, $v(x, \theta)=g(x, \theta)$ for $x \in \mathcal{J}$. Combining these observations shows that $g$ violates Assumption 5. Therefore, $x(\theta)$ is the unique optimal stopping threshold.

Berge's Maximum Theorem applied to the domain $[0, \bar{x}]$, guarantees that the correspondence associating to each $\theta$ the set of optimal stopping thresholds, starting from a given $x$, is upper hemicontinuous. This, combined with the established uniqueness of an optimal threshold implies continuity of $x(\theta)$.

\footnotetext{
${ }^{65}$ Quah and Strulovici (2012a) provide a signed-ratio condition for the sum of single crossing functions to be a single crossing function. Strict single crossing of $h(x, \theta)=\bar{h}(x, \theta)+\hat{h}(x, \theta)$ holds if $\bar{h}$ satisfies weak single crossing in $x$ and $\hat{h}$ is strictly increasing in $x$.

${ }^{66}$ In that case, continuing from $x>\hat{x}$ yields a flow payoff that is dominated by $r g(x)$ until $\hat{x}$ is reached. Choosing $\bar{x}$ high enough above $\hat{x}$ ensures that $\hat{x}$ is reached in a long enough time that the potential benefit from continuation, gained only after $\hat{x}$ is reached, is outweighed by the running cost incurred until that time.

${ }^{67} \theta$ is fixed and plays no role in this proof.
} 
For each $y$, let $w(x, \theta, y)$ denote the expected payoff, starting from $x$, if the agent uses the stopping region $[y,+\infty)$. Our assumptions imply that $w$ is differentiable with respect to $\theta$ for all $x$, with $w_{\theta}(x, \theta, x)=g_{\theta}(x, \theta)$, and that $w_{\theta}$ is continuous in $(\theta, y)$. References are provided in the Appendix, along with the proof of Proposition 8 .

Proposition 8 (Envelope Theorem) Under Assumption 5, the value function $v(x, \theta)$ is differentiable in $\theta$, for all $x$ Moreover, $v_{\theta}(x, \theta)=w_{\theta}(x, \theta, x(\theta))$.

Proposition 8 is used in the Appendix to derive a differential equation for $v_{\theta}$. Here, $f^{\prime}$ and $f^{\prime \prime}$ denote derivatives with respect to $x$; derivatives with respect to $\theta$ are denoted $f_{\theta}, g_{\theta}$, etc.

Proposition 9 For each $\theta$, let $\tau(\theta)=\inf \left\{t: X_{t} \geq x(\theta)\right\}$. Then,

$$
\begin{aligned}
v_{\theta}(x, \theta)= & E\left(\int_{0}^{\tau(\theta)} e^{-r t}\left(f_{\theta}\left(X_{t}, \theta\right)+\mu_{\theta}\left(X_{t}, \theta\right) v^{\prime}\left(X_{t}, \theta\right)+\sigma\left(X_{t}, \theta\right) \sigma_{\theta}\left(X_{t}, \theta\right) v^{\prime \prime}\left(X_{t}, \theta\right)\right) d t\right. \\
& \left.+e^{-r \tau(\theta)} g_{\theta}(x(\theta), \theta)\right)
\end{aligned}
$$

\subsection{Example: Option Value and Volatility}

Consider the case in which $\theta>0$ only affects the variance, linearly: $\sigma\left(X_{t}\right)=\tilde{\sigma}\left(X_{t}\right)+\theta$.

Corollary 4 Suppose that, for each $\theta, v(x, \theta)$ is convex on $[0, x(\theta)]$. Then, $v(x, \theta)$ is increasing in $\theta$ for $x<x(\theta)$ and $x(\theta)$ is nondecreasing in $\theta 69$

Proof. Equation (27) applied to this example yields, for $x<x(\theta)$,

$$
v_{\theta}(x, \theta)=E\left(\int_{0}^{\tau} e^{-r t} \sigma\left(X_{t}\right) v^{\prime \prime}\left(X_{t}, \theta\right) d t\right)
$$

which is positive by convexity of $v$. For $\tilde{\theta}>\theta$ and any $x<x(\theta)$, we have $v(x, \tilde{\theta}) \geq v(x, \theta)>g(x)$. This shows that $x(\tilde{\theta}) \geq x(\theta)$ and, hence, monotonicity of the threshold.

\footnotetext{
${ }^{68}$ The role of uniqueness underlying Proposition 8 may be understood as follows: if, for some parameter $\hat{\theta}$, it were optimal to stop at $\hat{x}_{1}$ or continue until $\hat{x}_{2}>\hat{x}_{1}$, and if $\hat{x}_{1}$ (resp. $\hat{x}_{2}$ ) were the only optimal threshold for $\theta<\hat{\theta}$ (resp. $\theta>\hat{\theta})$, then $v(x, \theta)$ would typically not be differentiable at $\hat{\theta}$, for $x<\hat{x}_{1}$ : its left derivative would be computed using the threshold $\hat{x}_{1}$, while its right derivative would be $\hat{x}_{2}$. Uniqueness of an optimal threshold guarantees that this situation cannot occur.

${ }^{69}$ It does not seem straightforward to derive this result using Jensen's inequality, even if $f$ and $g$ are both convex, because the stopping time is correlated with the state process.
} 


\section{Conclusion}

This paper provides unifying and self-contained arguments showing, under simple conditions, the smoothness of value functions and the existence of optimal strategies. These arguments can be extended to analyze situations in which the volatility vanishes over some subset of the state space, the control domain depends on the state, or control and stopping problems are combined. They can also be used to derive specific results in applications, such as bounding the number of switching points in finite multi-armed bandit problems, proving the uniqueness of an optimal threshold in optimal stopping problems, establishing effort monotonicity in a principal-agent model, or differentiability of the optimal strategy in growth or investment models.

The analysis has emphasized the role played by comparative statics for dynamic optimization problems. Single crossing conditions were used to prove the existence of an optimal control and to guarantee uniqueness of an optimal stopping threshold. This uniqueness, combined with an envelope theorem, was used to prove the smoothness of value functions in parameterized stopping problems. Envelope theorems were also helpful in applications to prove the differentiability of policy functions and the existence of an optimal control. This suggests that these tools, whose use is currently largely circumscribed to economic analysis, have a role to play in control theory, granting them a new, indirect role, for economics.

The assumption of a one-dimensional state space, while accounting for many economic models, is clearly restrictive. For example, we do not consider the case of a state that includes a time dimension or, in multi-armed bandit problems, the case in which each arm has a separate, independent state. Such extensions are special, as only one state evolves stochastically at any given time, which may be helpful in obtaining useful generalizations of the methods. More generally, however, there is a real need in economics for a better understanding of the properties of optimal policies and value functions with a multidimensional state space, both for qualitative analysis and for constructing explicit solutions.

\section{A Proof of Lemma 1}

Consider the function $\varphi$ defined on $\mathbb{R}$ by $\varphi(x)=|x|$ for $|x| \geq 1$ and $\varphi(x)=\frac{1}{2}\left(1+x^{2}\right)$ for $|x|<1$. As is easily checked, $\varphi$ is $C^{1}$ everywhere, $C^{2}$ except at -1 and 1 , and satisfies $\left|\varphi^{\prime}(x)\right| \leq 1$ and $|x| \leq \varphi(x) \leq|x|+1$ for all $x \in \mathbb{R}$ and $\left|\varphi^{\prime \prime}(x)\right| \leq 1$ for $x<1$ and $\varphi^{\prime \prime}(x)=0$ for $|x|>1$. By Itô's 
lemma 70 we have for any admissible control $A$, and $t \geq 0$

$$
\varphi\left(X_{t}^{A}\right)=\varphi(x)+\int_{0}^{t}\left(\mu\left(X_{s}^{A}, A_{s}\right) \varphi^{\prime}\left(X_{s}^{A}\right)+\frac{1}{2} \sigma^{2}\left(X_{s}^{A}, A_{s}\right) \varphi^{\prime \prime}\left(X_{s}^{A}\right)\right) d s+\int_{0}^{t} \sigma\left(X_{s}^{A}, A_{s}\right) \varphi^{\prime}\left(X_{s}^{A}\right) d B_{s} .
$$

Because $|\sigma(x, a)| \leq K^{\sigma}(1+|x|)$, the integrand of the stochastic integral is square integrable over $[0, t]{ }^{71}$ Therefore, the stochastic integral has zero mean. Taking expectations, and using the bounds on $\mu, \sigma$ and $\varphi$ we get, letting $\Phi_{t}=E \varphi\left(X_{t}^{A}\right)$,

$$
\Phi_{t} \leq \Phi(x)+\int_{0}^{t}\left(\left(K_{1}^{\mu}+K_{2}^{\mu} \Phi_{s}\right)+\frac{1}{2} \tilde{K}\right) d s
$$

where $\tilde{K}=\max _{(x, a) \in[-1,1]] \times \mathcal{K}} \sigma^{2}(x, a)$. By Gröwnwall's lemma, this implies that

$$
\Phi_{t} \leq\left(\Phi(x)+K_{X} t\right) \exp \left(K_{2}^{\mu} t\right)
$$

where $K_{X}=K_{1}^{\mu}+\tilde{K} / 2$. Since $\left|X_{t}\right| \leq \varphi\left(X_{t}\right)$ for all $t$, we conclude that $E\left|X_{t}\right| \leq(|x|+1+$ $\left.K_{X} t\right) \exp \left(K_{2}^{\mu} t\right)$ for all $t$. In particular, $E\left|X_{t}\right| e^{-r t}$ goes to zero as $t$ goes to infinity. The last claim of the lemma is straightforward to prove, using the linear growth condition on $f$.

\section{B Proof of Proposition 1}

\section{B.1 General Results on Initial Value Problems}

We start with two results pertaining to the existence of solutions to initial value problems (IVP) and their continuity with respect to the initial conditions. We start with some function $\bar{H}:(x, y) \mapsto$ $\bar{H}(x, y)$ defined on $\mathcal{X} \times \mathbb{R}^{n}$ and taking values in $\mathcal{R}^{n}$, which satisfies the following condition:

Condition 4 On any compact interval $\mathcal{I}$ of $\mathcal{X}$,

i) $|H(x, y)| \leq M(1+|y|)$

ii) $\left|H(x, y)-H\left(x, y^{\prime}\right)\right| \leq K\left|y-y^{\prime}\right|$,

iii) $H$ is continuous in $x$ for each $y$.

LEMma 4 If Condition 4 holds, the ordinary differential equation

$$
y^{\prime}(x)=H(x, y(x))
$$

with initial condition $y\left(x_{0}\right)=y_{0}$ has a unique continuously differentiable solution on $\mathcal{X}$, for any $x_{0} \in \mathcal{X}$ and $y_{0} \in \mathbb{R}^{n}$.

\footnotetext{
${ }^{70}$ Itô's lemma applies to any function that is $C^{1}$ and a.e. $C^{2}$.

${ }^{71}$ Precisely, square integrability follows from the inequality $\sigma^{2}\left(X_{s}^{A}, A_{s}\right) \leq 2\left(K^{\sigma}\right)^{2}\left(1+x^{2}\right)$, as well as a standard estimate on $E\left|X_{t}^{A}\right|^{2}$. See Krylov (1980), Corollary 6, p. 81.
} 
Let $y\left(x, y_{0}\right)$ denote the solution to 28 on $\mathcal{X}$ with initial condition $y\left(x_{0}\right)=y_{0}$.

Lemma 5 Given Condition $4 y\left(\cdot, y_{0}\right)$ is uniformly continuous in $y_{0}$.

The proofs are standard and omitted. For Lemma 4 see Hartman (2002, Theorem 1.1, p. 8) ${ }^{72}$ For Lemma 5, see Hartman (2002, Theorem 2.1, p. 94).

\section{B.2 Proof of Proposition 1: Bounded Case}

We specialize the results of Section B.1 to our setting: suppose that $y=(p, q)$ and $H(x, p, q)$ satisfies Condition 1. In this case, the function $\bar{H}(x,(p, q))=(q, H(x, p, q))$ satisfies Condition 4 . The proof of Proposition 1 is based on the "shooting method" (see, e.g., Bailey, 1962). The general intuition for the argument is as follows. We start from some initial conditions $(\underline{x}, \underline{v})$ and consider the solution $w$ to the IVP

$$
w^{\prime \prime}+H\left(x, w, w^{\prime}\right)=0
$$

subject to the initial conditions $w(\underline{x})=\underline{v}$ and $w^{\prime}(\underline{x})=s$. Given our assumptions on $H$, Lemma 4 guarantees that this IVP will have a unique, twice continuously differentiable solution. Lemma 5 guarantees that the solution continuously depends on the starting slope $s$. We can establish the existence of a solution to the boundary value problem (BVP) if we can show that it is always possible to pick the slope $s$ in such a way that at $\bar{x}$, the solution to the IVP will hit $\bar{v}$.

The proof relies on constructing a particular compact, convex subset of $(x, v)$-plane, ending with a vertical segment at $x=\bar{x}$ that contains $\bar{v}$. We then define a mapping between the initial slope $s$ of the solution to the IVP with initial value $w(\underline{x})=\underline{v}$ and initial slope $s$, and the "last" point at which it hits the boundary, and show that the mapping is onto. That property then proves the existence of an initial slope such that the solution hits the value $\bar{v}$ at $\bar{x}$.

Lemma 6 There exist positive constants $K_{1}, K_{2}$ such that the functions $b_{1}(x)=-K_{1}-K_{2}|x|$ and $b_{2}(x)=K_{1}+K_{2}|x|$ satisfy the inequalities

$$
\begin{aligned}
& b_{1}^{\prime \prime}+H\left(x, b_{1}, b_{1}^{\prime}\right)>0 \\
& b_{2}^{\prime \prime}+H\left(x, b_{2}, b_{2}^{\prime}\right)<0
\end{aligned}
$$

for all $x \neq 0$, and the boundary constraints $\underline{v} \in\left(b_{1}(\underline{x}), b_{2}(\underline{x})\right)$ and $\bar{v} \in\left(b_{1}(\bar{x}), b_{2}(\bar{x})\right)$.

\footnotetext{
${ }^{72}$ That theorem establishes local existence. The growth condition, i), guarantees that the solution can be extended to the entire domain $\mathcal{I}$.
} 
Proof. We have for $x \neq 0$ and $K_{1}, K_{2}$ satisfying Condition 3 ,

$$
\left.b_{2}^{\prime \prime}(x)+H\left(x, b_{2}(x), b_{2}^{\prime}(x)\right)=H\left(x, K_{1}+K_{2}|x|\right), K_{2} \operatorname{sgn}(x)\right),
$$

which is strictly negative. The argument for $b_{1}$ is analogous. The boundary constraints are clearly satisfied provided that $K_{1}$ and $K_{2}$ are large enough.

Lemma 7 There exist $s_{1}$ and $s_{2}$ such that the solution to IVP (29) hits $b_{2}$ for all initial slopes $s \geq s_{2}$ and $b_{1}$ for all initial slopes $s \leq s_{1}$.

Proof. By suitably translating the problem, we can without loss assume that $\underline{x}=\underline{v}=073$ We wish to show that for high enough initial slopes $s$, the solution $w_{s}$ hits $b_{2}$. Consider the auxiliary IVP

$$
u^{\prime \prime}+K u^{\prime}+H(x, u(x), 0)+\varepsilon=0
$$

subject to $u(0)=0$ and $u^{\prime}(0)=s$, where $K$ is the Lipschitz constant of $H$ and $\varepsilon$ is a positive constant. We will show that, for $s$ high enough, $u$ is strictly increasing on $[0, \bar{x}]$, with a derivative that is bounded below by a linear function of $s$. For fixed $s$, let $\tilde{x}>0$ denote the first time that $u^{\prime}(x)=0$. On $[0, \tilde{x}]$, we have $u(x) \geq 0$. By Condition 2 , we have $H(x, u(x), 0) \leq H(x, 0,0)$ on that domain, and

$$
u^{\prime \prime}(x)+K u^{\prime}(x)+M \geq 0,
$$

where $M=\max _{x \in[0, \bar{x}]}|H(x, 0,0)|+\varepsilon>0$. Applying Grönwall's inequality to the function $g(x)=$ $-u^{\prime}(x)-M / K$, which satisfies the inequality $g^{\prime}(x) \leq-K g(x)$ on $[0, \tilde{x}]$, we conclude that

$$
u^{\prime}(x) \geq[s+M / K] \exp (-K x)-M / K
$$

on that domain. This implies that $\tilde{x}$ is bounded below by

$$
\frac{1}{K} \log \left(\frac{s+M / K}{M / K}\right)
$$

which exceeds $\bar{x}$, for $s$ high enough. Moreover, the lower bound on $u^{\prime}$ also implies that $u$ hits $b_{2}$ for $s$ large enough and does not cross it again before $\bar{x}$ is reached.

To conclude the proof, we will show that the IVP solution $w$ is above $u$ for any fixed $s$. The Lipschitz property of $H$ in its last argument implies that, for all $x, u, u^{\prime}$,

$$
-K u^{\prime} \leq H(x, u, 0)-H\left(x, u, u^{\prime}\right) .
$$

\footnotetext{
${ }^{73}$ The translation is obtained by letting $\bar{w}(x)=w(x-\underline{x})-\underline{v}$ and $\bar{H}\left(x, w, w^{\prime}\right)=H\left(x-\underline{x}, w+\underline{v}, w^{\prime}\right)$. $\bar{H}$ inherits the Lipschitz and monotonicity properties of $H$, as is easily checked.
} 
From the definition of $u$, this implies that

$$
u^{\prime \prime}(x)+H\left(x, u(x), u^{\prime}(x)\right) \leq-\varepsilon<0
$$

for all $x$. This implies that $w$, the solution to the IVP, lies above $u$, by the following argument. At $x=0, u$ and $w$ have the same starting values and slopes, but $u$ has a lower second derivative, by at least $\varepsilon$, which implies that $u^{\prime}<w^{\prime}$ in a right neighborhood of 0 . We will show that $u^{\prime}<w^{\prime}$ for all $x$ in $(0, \bar{x}]$ and, therefore, that $u<w$ on that domain. Suppose by contradiction that there exists an $x>0$ such that $u^{\prime}(x)=w^{\prime}(x)$, and let $\tilde{x}$ be the first such point. Necessarily, $u(\tilde{x})<w(\tilde{x})$. Moreover, we have

$$
u^{\prime \prime}(\tilde{x})<-H\left(\tilde{x}, u(\tilde{x}), u^{\prime}(\tilde{x})\right) \leq-H\left(\tilde{x}, w(\tilde{x}), w^{\prime}(\tilde{x})\right)=w^{\prime \prime}(\tilde{x}),
$$

where the second inequality is guaranteed by Condition 2. This contradicts the fact that $u^{\prime}$ crosses $w^{\prime}$ from below at $\tilde{x}$.

We can finally prove Proposition 1 . Let

$$
B=\left\{(x, v) \mid b_{1}(x)=v \text { or } b_{2}(x)=v\right\} \cup\left[\left(\bar{x}, b_{1}(\bar{x})\right),\left(\bar{x}, b_{2}(\bar{x})\right)\right] \subset \mathbb{R}^{2} .
$$

$B$ consists of the graph of the functions $b_{1}$ and $b_{2}$ on $\mathcal{X}$, along with the vertical segment joining the endpoints of these graphs at $\bar{x}$. We also define the function $\mathcal{H}:\left[s_{1}, s_{2}\right] \rightarrow \mathbb{R}^{2}$ as the last hitting point of $B$ for the solution of the IVP with slope $s$. This function is clearly well defined: if a solution does not cross $b_{1}$ or $b_{2}$ before $\bar{x}$, it has to hit the vertical segment joining $b_{1}(\bar{x})$ and $b_{2}(\bar{x})$. From Lemma 7. $\mathcal{H}(s)$ is on the graph of $b_{2}$ for $s$ large and on the graph of $b_{1}$ for $s$ small (for example, (30) shows that, for $s$ large enough, $u$ cannot cross $b_{2}$ again after hitting it once). Moreover, $\mathcal{H}$ cannot jump from the graph of $b_{2}$ to the graph of $b_{1}$ as $s$ changes, because Lemma 6 implies, for example, that if $w$ crosses $b_{2}$, it stays above $b_{2}$ for all $x$ beyond the crossing point. ${ }^{74}$ and hence cannot hit $b_{1}$. Therefore, $\mathcal{H}$ must connect the upper and lower bounds of $B$ as $s$ goes down. Finally, Lemma 5 implies that $\mathcal{H}$ is continuous at any point $s$ for which $\mathcal{H}(s)$ lies on the vertical segment. This shows that $\mathcal{H}(s)$ must take all values on that segment as it connects the graphs of $b_{2}$ and $b_{1}$. Since $(\bar{x}, \bar{v})$ belongs to that segment, this proves existence of a solution that solves the BVP.

\section{B.3 Proof of Proposition 1; Unbounded Domain}

We now prove Proposition 1 when $\mathcal{X}$ is unbounded, so that $\underline{x}=-\infty$ and/or $\bar{x}=+\infty$. Precisely, we establish the existence of a function $v$ which satisfies

$$
w^{\prime \prime}=H\left(x, w, w^{\prime}\right)
$$

\footnotetext{
${ }^{74}$ The proof of this result is similar to the proof that $w$ stays above $u$ in Lemma 7 showing that $w^{\prime} \geq b_{2}^{\prime}$ after the crossing point, and exploits the inequality $b_{2}^{\prime \prime}+H\left(x, b_{2}, b_{2}^{\prime}\right)<0$.
} 
and $|w(x)| \leq K_{v}(1+|x|)$ on $\mathcal{X}$, where $K_{v}$ is a positive constant. The arguments of this section are based on Schrader (1969). For expositional simplicity we focus on the case in which $\mathcal{X}=\mathbb{R}$. The case in which either $\underline{x}$ or $\bar{x}$ is finite follows easily from that argument.

Throughout the argument we fix a pair $K_{1}, K_{2}$ of constants that satisfy Condition 3 , and let $b_{1}, b_{2}$ denote the bounds constructed from these constants in Lemma 6. From Section B.2, we know that the BVP will have a unique $C^{2}$ solution for any finite interval $[\underline{\chi}, \bar{\chi}]$ and boundary conditions $w(\underline{\chi})=\underline{v}$ and $w(\bar{\chi})=\bar{v}$ that are contained between $b_{1}$ and $b_{2}$. Further, we know that the solution satisfies $-K_{v}(1+|x|) \leq w(x) \leq K_{v}(1+|x|)$ on $[\underline{\chi}, \bar{\chi}]$, where $K_{v}=\max \left\{K_{1}, K_{2}\right\}$ does not depend on the particular interval chosen.

We define a sequence of boundary value problems such that Equation (31) holds on $\left[\underline{x}_{n}, \bar{x}_{n}\right]$ and with boundary conditions $w\left(\underline{x}_{n}\right)=\underline{v}_{n}$ and $w\left(\bar{x}_{n}\right)=\bar{v}_{n}$ for some values $\underline{v}_{n}, \bar{v}_{n}$ in $\left(b_{1}\left(\underline{x}_{n}\right), b_{2}\left(\underline{x}_{n}\right)\right)$ and $\left(b_{1}\left(\bar{x}_{n}\right), b_{2}\left(\bar{x}_{n}\right)\right)$, respectively, and let $\underline{x}_{n}$ and $\bar{x}_{n}$ tend to $-\infty$ or $+\infty$, respectively.

Let $w_{n}$ denote the solution to the $n^{\text {th }}$ BVP. In the following, we use the Arzelà-Ascoli theorem and show that this procedure indeed yields a solution. For this, we need to prove that the derivatives of $w_{n}$ are equicontinuous. This is based on the following comparison lemma 75

LEMma 8 Let $\phi$ denote a nonnegative, continuous function on $\mathbb{R}_{+}$, such that

$$
\int_{0}^{\infty} \frac{s}{\phi(s)} d s=\infty
$$

and let $R, \tilde{x}$ denote two strictly positive constants. Then, there exists a number $M$ such that if $w(x)$ is $C^{2}$ on $[0, \bar{x}]$ with $\bar{x}>\tilde{x}$ and satisfies $|w(x)| \leq R$ and $\left|w^{\prime \prime}(x)\right| \leq \phi\left(\left|w^{\prime}(x)\right|\right)$, then $\left|w^{\prime}(x)\right| \leq M$ on $[0, \bar{x}]$. The constant $M$ depends only on $R, \phi$ and $\tilde{x}$.

For any bounded domain $\mathcal{X}_{0}=[\underline{\chi}, \bar{\chi}]$ and any solution $w$ to the BVP on that domain with end values between $b_{1}$ and $b_{2}$, we have

$$
\left|w^{\prime \prime}(x)\right|=\mid H\left(x, w(x), w^{\prime}(x)|\leq| H(x, w(x), 0)|+K| w^{\prime}(x)|\leq \bar{K}+K| w^{\prime}(x) \mid\right.
$$

where $K$ is the Lipschitz constant of $H$ over $\mathcal{X}_{0}$ and where the constant $\bar{K}$ comes from the boundedness of $w$ (which is contained between $b_{1}$ and $b_{2}$ ) and continuity of $H(\cdot, \cdot, 0)$ on the compact domain $\mathcal{X}_{0}$.

Since $\phi(x)=\bar{K}+K x$ satisfies (32) and $w$ is bounded by the functions $b_{1}$ and $b_{2}$, Lemma 8 implies that each $w_{n}^{\prime}$ is bounded on the compact domain $\mathcal{X}_{0}$, and that the bound is uniform over $n 7^{76}$ Moreover, (33) implies that the second derivatives of $w_{n}$ are also uniformly bounded on $\mathcal{X}_{0}$.

\footnotetext{
${ }^{75}$ See Hartman (2002), p. 428.

${ }^{76}$ More precisely, we can apply the Lemma to the function $\bar{w}(x)=w(x-\underline{\chi})$, so as to have the 0 origin that is assumed in the Lemma.
} 
We now use the following diagonalization procedure. Consider a finite domain $\left[\underline{x}_{1}, \bar{x}_{1}\right]$. We have seen that the functions $w_{n}, w_{n}^{\prime}$ and $w_{n}^{\prime \prime}$ are bounded on $\left[\underline{x}_{1}, \bar{x}_{1}\right]$, uniformly in $n$. By Arzelà-Ascoli's theorem, there exists a subsequence such that $w_{n}$ converges uniformly to a $C^{1}$ function $\tilde{w}_{1}$ on $\left.\left[\underline{x}_{1}, \bar{x}_{1}\right]\right]^{77}$ Moreover, the second derivatives $\left\{w_{n}^{\prime \prime}\right\}_{n \in \mathbb{N}}$ are also equicontinuous, because they satisfy $w_{n}^{\prime \prime}(x)=H\left(x, w_{n}(x), w_{n}^{\prime}(x)\right)$ with $H$ continuous and $w_{n}$ and $w_{n}^{\prime}$ equicontinuous. This implies that there is a subsequence of $w_{n}$ that converges uniformly to a $C^{2}$ function $\tilde{w}_{1}$ on $[\underline{x}, \bar{x}]$. This also implies that the limit satisfies $\tilde{w}_{1}^{\prime \prime}(x)=-H\left(x, \tilde{w}_{1}(x), \tilde{w}_{1}^{\prime}(x)\right)$. By construction, $b_{1}(x) \leq w_{n}(x) \leq b_{2}(x)$ on $\left[\underline{x}_{1}, \bar{x}_{1}\right]$ and, therefore, $\tilde{w}_{1}$ is also contained between $b_{1}$ and $b_{2}$.

To conclude, take the finite domain $\left[\underline{x}_{2}, \bar{x}_{2}\right] \supset\left[\underline{x}_{1}, \bar{x}_{1}\right]$. Iterating the last argument ${ }^{78}$ there exists a subsequence of the first subsequence for which $w_{n}$ converges uniformly to a limit function $\tilde{w}_{2}$ on $\left[\underline{x}_{2}, \bar{x}_{2}\right]$. The functions $\tilde{w}_{1}$ and $\tilde{w}_{2}$ are equal on $\left[\underline{x}_{1}, \bar{x}_{1}\right]$. Proceeding iteratively, we can cover the entire domain $\mathcal{X}$. The function $w$ defined by $w(x)=\tilde{w}_{k}(x)$ for $x \in\left[\underline{x}_{k}, \bar{x}_{k}\right] \backslash\left[\underline{x}_{k-1}, \bar{x}_{k-1}\right]$, solves the BVP and is bounded by $b_{1}$ and $b_{2}$ and hence also satisfies $|w(x)| \leq K_{v}(1+|x|)$.

\section{Proofs of Section 5}

Lemma 9 Consider $v_{\tilde{s}}$ and $v_{s}$, two solutions to the IVP with starting slopes $\tilde{s}>s$ on an interval $\left[x_{1}, x_{2}\right]$ which both satisfy $v_{\tilde{s}}\left(x_{1}\right)=v_{s}\left(x_{1}\right)=v_{1}$. Then, $v_{\tilde{s}}(x)>v_{s}(x)$ for all $x \in\left(x_{1}, x_{2}\right]$.

Proof. Let $\hat{x}=\inf \left\{x: v_{\tilde{s}}^{\prime}(x) \leq v_{s}^{\prime}(x)\right\}$. Note that $\hat{x}>\underline{x}$ because $v_{\tilde{s}}^{\prime}(\underline{x})>v_{s}^{\prime}(\underline{x})$ and both $v_{\tilde{s}}$ and $v_{s}$ are $C^{2}$. By construction, $v_{\tilde{s}}(\hat{x})>v_{s}(\hat{x})$. Since both solutions satisfy the equation

$$
v^{\prime \prime}(x)+\frac{1}{\frac{1}{2} \sigma(x)^{2}}\left(-r v(x)+f(x)+\mu(x) v^{\prime}(x)\right)=0
$$

we have

$$
\begin{aligned}
v_{\tilde{s}}^{\prime \prime}(\hat{x}) & =\frac{1}{\frac{1}{2} \sigma(\hat{x})^{2}}\left(r v_{\tilde{s}}(\hat{x})+f(\hat{x})+\mu(\hat{x}) v_{\tilde{s}}^{\prime}(\hat{x})\right) \\
& >\frac{1}{\frac{1}{2} \sigma(\hat{x})^{2}}\left(r v_{s}(\hat{x})+f(\hat{x})+\mu(\hat{x}) v_{s}^{\prime}(\hat{x})\right)=v_{s}^{\prime \prime}(\hat{x})
\end{aligned}
$$

Since $v_{\tilde{s}}^{\prime}(x)$ must hit $v_{s}^{\prime}(x)$ from above as $x$ reaches $\hat{x}$, we obtain a contradiction.

\footnotetext{
${ }^{77}$ More precisely, we use the following version: any sequence of $C^{1}$ functions that have equicontinuous and uniformly bounded derivatives, and are uniformly bounded at one point, has a subsequence that converges uniformly to a $C^{1}$ function. Here, equicontinuity of the derivatives is guaranteed by the uniform bound on the second derivative.

${ }^{78}$ Note that the bounds for the domains $\left[\underline{x}_{2}, \bar{x}_{2}\right]$ and $\left[\underline{x}_{1}, \bar{x}_{1}\right]$ are different. However, since we are fixing the domain, we are still able to obtain a convergent subsequence.
} 


\section{C.1 End of the proof of Theorem 4}

To conclude the proof, we need to show the result when $x$ is such that $v(x)=g(x)$, but $x$ is an accumulation point of stopping and continuation regions, on either its right side or its left side, or both.

Without loss of generality, we set $x=0$ and prove that $v_{r}(0)=g^{\prime}(0)$, where $v_{r}$ is the right derivative of $v$ at 0 . We wish to show that $\lim _{\eta \downarrow 0}(v(\eta)-v(0)) / \eta$ converges to $g^{\prime}(0)$. Consider any $\eta>0$. The difference $v(\eta)-v(0)$ is either equal to $g(\eta)-g(0)$, if $\eta$ belongs to $\mathcal{Y}$, or else $\eta$ belongs to some interval $\mathcal{Z}_{i}$ close to 0 . Let $y$ denote the lower bound of $\mathcal{Z}_{i}$. By twice differentiability of $v$ on $(y, \eta)$, and because the right derivative of $v$ at $y$ is equal to $g^{\prime}(y)$, we have $v(\eta)=v(y)+g^{\prime}(y)(\eta-y)+$ $\frac{1}{2} v_{r}^{\prime \prime}\left(z_{1}\right)(\eta-y)^{2}$ for some $z_{1} \in(y, \eta)$. Since $v(y)=g(y)$, we have $g(\eta)=v(y)+g^{\prime}\left(z_{2}\right)(\eta-y)$ for some $z_{2} \in(y, \eta)$. Therefore, $v(\eta)=g(\eta)+\left(g^{\prime}(y)-g^{\prime}\left(z_{2}\right)\right)(\eta-y)+\frac{1}{2} v_{r}^{\prime \prime}\left(z_{1}\right)(\eta-y)^{2}$, and

$$
\frac{v(\eta)-v(0)}{\eta}=\frac{g(\eta)-g(0)}{\eta}+1_{\eta \notin \mathcal{Y}} \frac{\eta-y}{\eta}\left(g^{\prime}(y)-g^{\prime}\left(z_{2}\right)+\frac{1}{2} v_{r}^{\prime \prime}\left(z_{1}\right)(\eta-y)\right) .
$$

Taking the limit as $\eta$ goes to zero yields the result, if we can show that $g^{\prime}(y)-g^{\prime}\left(z_{2}\right)+\frac{1}{2} v_{r}^{\prime \prime}\left(z_{1}\right)(\eta-y)$ converges to zero as $\eta \rightarrow 0$. The first two terms cancel each other in the limit, as they both converge to $g^{\prime}(0)$ (since $g$ is $C^{1}$ ). The last term converges to 0 if we can show that $v^{\prime \prime}(\cdot)$ is uniformly bounded on all the intervals $\mathcal{Z}_{i}$ in a neighborhood of 0 . This uniform bound is guaranteed by Lemma 8 (Section B.3), which guarantees a uniform upper bound on $\left|v^{\prime}\right|$ and on $\left|v^{\prime \prime}\right|$.79

Continuity of $v^{\prime}$ is shown by a similar argument. For any $\varepsilon>0$, there exists $\bar{\eta}(\varepsilon)$ such that $\left|g^{\prime}(\eta)-g^{\prime}(0)\right| \leq \varepsilon / 2$ for all $\eta \leq \bar{\eta}(\varepsilon)$. As was mentioned earlier, $v^{\prime \prime}$ is uniformly bounded on the interval $[x, x+\bar{\eta}(\varepsilon)] \cap \cup_{i \in \mathcal{I}} \mathcal{Z}_{i}$, by some constant $M$. Let $\eta(\varepsilon)=\min \{\bar{\eta}(\varepsilon), \varepsilon / M\}$. Consider any $\eta<\eta(\varepsilon)$. If $\eta \in \mathcal{Y}$, then $v^{\prime}(\eta)=g^{\prime}(\eta)$ and $\left|v^{\prime}(\eta)-v^{\prime}(0)\right|<\varepsilon / 2$. Otherwise we have, using the variable $y$ introduced earlier in the proof,

$$
v^{\prime}(\eta)=v^{\prime}(y)+v^{\prime \prime}\left(z_{3}\right)(\eta-y)=g^{\prime}(y)+v^{\prime \prime}\left(z_{3}\right)(\eta-y)
$$

for some $z_{3} \in(y, \eta)$. This implies that

$$
\left|v^{\prime}(\eta)-v^{\prime}(0)\right|<\left|g^{\prime}(y)-g^{\prime}(0)\right|+M \eta<\varepsilon
$$

Proceeding similarly to the left of $x$, we conclude that $v^{\prime}$ is continuous at $x$.

\footnotetext{
${ }^{79}$ More precisely, the solution $w_{i}$ to the $\mathrm{BVP}$ on $\mathcal{Z}_{i}$ with endpoints $\left[\underline{x}_{i}, \bar{x}_{i}\right]$ can be extended to a solution on the domain $\mathcal{Z}_{i}^{\prime}=\left[\underline{x}_{i}, \max \left\{\underline{x}_{i}+\tilde{x}, \bar{x}_{i}\right\}\right]$, for some $\tilde{x}>0$ that is independent of $i$, by considering the IVP with domain $\mathcal{Z}_{i}^{\prime}$ and keeping the same initial value and slope at $\underline{x}_{i}$. That solution has uniformly bounded first derivative, by Lemma 8 and hence also a uniformly bounded second derivative (see (33)). The uniform bounds do not depend on $i$ : they only depend on $\tilde{x}$, an upper bound on $v$, and the fact that $\left|w^{\prime \prime}(x)\right|=\left|\frac{1}{\frac{1}{2} \sigma(x)^{2}}\left(-r w(x)+f(x)+\mu(x) w^{\prime}(x)\right)\right| \leq K_{1}+K_{2}\left|w^{\prime}(x)\right|$ for some constants $K_{1}, K_{2}$.
} 


\section{Proofs for the Multi-Armed Bandit Application}

Lemma 10 The value function $v$ is convex in the belief $x$.

Proof. The argument is standard and replicated for completeness. For any admissible control process $A$, we have

$$
v(x, A)=E\left[\int_{0}^{\infty} e^{-r t} \pi\left(A_{t}, \tilde{\theta}\right) d t \mid x\right]
$$

By definition, any admissible process $A$ is adapted to the observation filtration, which, conditional on the true state $\tilde{\theta}$ does not depend on the probability $x$. Therefore, we have

$$
v(x, A)=x E\left[\int_{0}^{\infty} e^{-r t} \pi\left(A_{t}, \theta_{H}\right) d t \mid \theta_{H}\right]+(1-x) E\left[\int_{0}^{\infty} e^{-r t} \pi\left(A_{t}, \theta_{L}\right) d t \mid \theta_{L}\right],
$$

which shows that $v(x, A)$ is linear in $x$. For $x_{1}<x_{2}$ and $\lambda \in(0,1)$, let $x=\lambda x_{1}+\left(1-\lambda x_{2}\right)$. We have, letting $A^{*}$ denote an optimal process given the initial belief $x{ }^{80}$

$$
v(x)=v\left(x, A^{*}\right)=\lambda v\left(x_{1}, A^{*}\right)+(1-\lambda) v\left(x_{2}, A^{*}\right) \leq \lambda v\left(x_{1}\right)+(1-\lambda) v\left(x_{2}\right),
$$

where the second equality comes from the linearity of $v(\cdot, A)$ in $x$, and the inequality comes from the definition of the $v\left(x_{i}\right)$ 's.

Proposition 10 With finitely many arms, the are finitely many cutoffs, and the number of cutoffs is bounded by twice the number of arms.

Proof. From Theorem 1, the value function solves everywhere the HJB equation, which may be rewritten as

$$
r v(x)=\max _{i \in \mathcal{K}}\left\{x \Delta f(i)+f_{L}(i)+v^{\prime \prime}(x) \frac{1}{2}(x(1-x))^{2} \xi^{2}(i)\right\}
$$

where $\Delta f(i)=f_{H}(i)-f_{L}(i)$. If $v^{\prime \prime}(x)=0$ for some $x \in(0,1)$, then $v(x)=\frac{x \Delta f(i)+f_{L}(i)}{r}$ for some $i$, which means that playing $i$ forever is optimal and there is no cutoff. Thus, we focus on the case $v^{\prime \prime}(x)>0$ for all $x \in(0,1)$.

Rewriting the HJB equation, we have

$$
v^{\prime \prime}(x)=\min _{i \in \mathcal{K}} \frac{r v(x)-x \Delta f(i)-f_{L}(i)}{\frac{1}{2}(x(1-x))^{2} \xi^{2}(i)} .
$$

Moreover, letting

$$
w_{i}(x)=\frac{r v(x)-x \Delta f(i)-f_{L}(i)}{\xi^{2}(i)}
$$

\footnotetext{
${ }^{80}$ We do not need to assume the existence of an optimum: the argument is easily adapted by taking a sequence of controls $A_{n}$ such that $v\left(x, A_{n}\right)$ converges to $v(x)$ as $n \rightarrow \infty$.
} 
Arm $i$ maximizes (34) if and only if $w_{i}(x) \leq w_{j}(x)$ for all $j$ in $\mathcal{K}$.

We have

$$
\left(w_{i}-w_{j}\right)^{\prime \prime}(x)=r v^{\prime \prime}(x)\left(\frac{\xi^{2}(j)-\xi^{2}(i)}{\xi^{2}(i) \xi^{2}(j)}\right) .
$$

Lemma 10 therefore implies that either $w_{i}-w_{j}$ or $w_{j}-w_{i}$ is convex. If $w_{i}-w_{j}$ is linear, either the arms are identical, in which case they can be treated as a single arm, or $w_{i}-w_{j}$ crosses zero at most once. Otherwise, $w_{i}-w_{j}$ is strictly convex or strictly concave, since $v^{\prime \prime}$ is strictly positive, and crosses zero at most twice. Since the number of arms is finite, this implies that the set $\mathcal{Z}_{i}=\left\{x: w_{i}(x)>w_{j}(x) \quad \forall j \neq i\right\}$ consists of finitely many open intervals, and that the set $\mathcal{Z}$ of $x \in[0,1]$ such that $\arg \max _{i}\left\{w_{i}(x)\right\}$ is not a singleton is finite. This shows that any selection of maximizers of the HJB equation has finitely many switches, yielding a volatility that has bounded variation. Theorem 2 then implies that such selection is optimal. It also implies that the (essentially unique) optimal strategy has finitely many cutoffs. Because any two arms can only change dominance twice, this also shows that the number of switching points is bounded by twice the number of arms: if an arm is strictly optimal in two disjoint regions, then any arm that is strictly optimal in a region contained between these two regions can only be optimal within these two regions. The result then follows by an easy induction argument.

Proposition 11 A given arm may be used over disjoint intervals of beliefs.

Proof. Consider a four-armed setting with the following characteristics. Arms 1 and 2 have symmetric payoffs around the belief $1 / 2: f_{1}(x)=f_{2}(1-x)$, with $f_{1}(0)>f_{1}(1)$, and have the same low signal-to-noise ratio $\xi(1)=\xi(2)=\varepsilon>0$. The payoffs $f_{3}$ and $f_{4}$ are independent ${ }^{81}$ of $x$, with $f_{1}(0)>f_{3}>f_{4}$ and $\min \{\xi(3), \xi(4)\}>\varepsilon$.

Notice that, for extreme beliefs, either Arm 1 or 2 is used. Moreover, because Arm 1 is optimal for very low beliefs whereas Arm 2 is optimal for very high ones, the value of information is strictly positive in this problem.

It suffices to show that Arms 3 and 4 are both chosen on a positive domain. By symmetry, this will imply that one arm is chosen on both sides (the one that is not used at $x=1 / 2$ ) ${ }^{82}$ Consider, first, the case in which only Arms 1,2, and 3 are available. Because Arms 1 and 2 have low signal to noise ratio, using Arm 3 is optimal for intermediate beliefs, provided that $f_{3}$ is high enough (but still below $\left.f_{1}(0)\right)$. We now add in Arm 4 , which has a low payoff. We will gradually increase its signal

\footnotetext{
${ }^{81}$ Thus, for simplicity, Arms 3 and 4 are informative despite having state-independent payoffs. It is easy to slightly perturb those payoffs to introduce payoff dependence and preserve the qualitative shape of the choice intervals.

${ }^{82}$ If $x=1 / 2$ was a cutoff, then both Arms 3 and 4 are used on each side of $x=1 / 2$, which also proves the proposition.
} 
to noise ratio, $\xi(4)$. For $\xi(4)<\xi(3)$, Arm 4 is dominated by Arm 3 both in terms of payoff and informativeness, and thus not used at all. For $\xi(4)$ arbitrarily high, using Arm 4 reveals the state of the world almost instantaneously, which is clearly optimal for intermediate beliefs. Therefore, there must exist a threshold $\xi_{4}^{*}>\xi(3)$ above which it becomes strictly optimal to use Arm 4 around some belief $x^{*}$. At $\xi_{4}^{*}, v_{4}-v_{3}$ has a local maximum at $x^{*}$, which has to be in the interior of $[0,1]$. From the proof of Proposition 10, this implies that $v_{4}-v_{3}$ is strictly concave and that $x^{*}$ is the unique global maximum. This implies that $v_{3}(x)>v_{4}(x)$ for all $x \neq x^{*}$ and, since it is always optimal to use either Arm 3 or Arm 4 on an interval of strictly positive measure, this implies that $v_{3}\left(x^{\prime}\right)>\max \left\{v_{1}\left(x^{\prime}\right), v_{2}\left(x^{\prime}\right), v_{4}\left(x^{\prime}\right)\right\}$ on some interval $\mathcal{Z}^{\prime}$ of positive measure. By increasing $\xi(4)$ slightly above $\xi_{4}^{*}$, Arm 4 becomes strictly optimal around $x^{*}$ (by definition of $x^{*}$ ), while Arm 3 remains strictly optimal on $\mathcal{Z}^{\prime}$, which proves the claim.

\section{E Proofs for Section 6 (Parameterized Stopping Problems)}

\section{E.1 Proof of Proposition 8}

Suppose that $x<x(\theta)$. Fixing any parameter $\theta$ and stopping threshold $\hat{x}, w(x, \theta, \hat{x})$ solves the dynamic equation

$$
r w(x, \theta, \hat{x})=f(x, \theta)+\mu(x, \theta) w^{\prime}(x, \theta, \hat{x})+\frac{1}{2} \sigma^{2}(x, \theta) w^{\prime \prime}(x, \theta, \hat{x})
$$

for all $x<\hat{x}$, and satisfies the boundary conditions $w(0, \theta)=0$ and $w(\hat{x}, \theta)=g(\hat{x}, \theta)$. Theorem 3.1 , p. 95 in Hartman (2002) implies that $w(x, \theta, \hat{x}), w^{\prime}(x, \theta, \hat{x})$ and $w^{\prime \prime}(x, \theta, \hat{x})$ are all differentiable with respect to $\theta$ for $x<\hat{x}$ and that the derivative $w_{\theta}(x, \theta, \hat{x})$ satisfies

$$
\begin{aligned}
r w_{\theta}(x, \theta, \hat{x})= & f_{\theta}(x, \theta)+\mu_{\theta}(x, \theta) w^{\prime}(x, \theta, \hat{x})+\sigma(x, \theta) \sigma_{\theta}(x, \theta) w^{\prime \prime}(x, \theta, \hat{x}) \\
& +\mu(x, \theta) w_{\theta}^{\prime}(x, \theta, \hat{x})+\frac{1}{2} \sigma^{2}(x, \theta) w_{\theta}^{\prime \prime}(x, \theta, \hat{x}) \\
= & h(x, \theta, \hat{x})+\mu(x, \theta) w_{\theta}^{\prime}(x, \theta, \hat{x})+\frac{1}{2} \sigma^{2}(x, \theta) w_{\theta}^{\prime \prime}(x, \theta, \hat{x})
\end{aligned}
$$

for all $x<\hat{x}$, where $h(x, \theta)=f_{\theta}(x, \theta)+\mu_{\theta}(x, \theta) w^{\prime}(x, \theta, \hat{x})+\sigma(x, \theta) \sigma_{\theta}(x, \theta) w^{\prime \prime}(x, \theta, \hat{x})$, with boundary conditions $w_{\theta}(0, \theta, \hat{x})=0$ and $w_{\theta}(\hat{x}, \theta, \hat{x})=g_{\theta}(\hat{x}, \theta)$.

Theorem 2.1 p. 94 and Corollary 3.3 p. 99, in Hartman (2002) applied to Equation (36) show that for any $x, w_{\theta}(x, \theta, \hat{x})$ is continuous in $(\theta, \hat{x})$. By Proposition 7, the maximizing threshold $x(\theta)$ is unique, and thus Corollary 4, part iii) of Milgrom and Segal (2002) implies that $v(x, \theta)=w(x, \theta, x(\theta))$ is differentiable in $\theta$, and that its derivative is equal to $w_{\theta}(x, \theta, x(\theta))$. 


\section{E.2 Proof of Proposition 9}

Differentiating the equation $v(x, \theta)=w(x, \theta, x(\theta))$ for $x<x(\theta)$ yields $v^{\prime}(x, \theta)=w^{\prime}(x, \theta, x(\theta))$ and $v^{\prime \prime}(x, \theta)=w^{\prime \prime}(x, \theta, x(\theta))$. Similarly, Proposition 8 has established that $v_{\theta}(x, \theta)=w_{\theta}(x, \theta, x(\theta))$. Differentiating this equation with respect to $x$ for $x<x(\theta)$ yields $v_{\theta}^{\prime}(x, \theta)=w_{\theta}^{\prime}(x, \theta, x(\theta))$ and $v_{\theta}^{\prime \prime}(x, \theta)=w_{\theta}^{\prime \prime}(x, \theta, x(\theta))$. Combining these equations with 36 yields

$$
r v_{\theta}(x, \theta)=h(x, \theta)+\mu(x, \theta) v_{\theta}^{\prime}(x, \theta)+\frac{1}{2} \sigma^{2}(x, \theta) v_{\theta}^{\prime \prime}(x, \theta),
$$

where $h(x, \theta)=f_{\theta}(x, \theta)+\mu_{\theta}(x, \theta) w^{\prime}(x, \theta, \hat{x})+\sigma(x, \theta) \sigma_{\theta}(x, \theta) w^{\prime \prime}(x, \theta, \hat{x})$. We also have the boundary conditions $v_{\theta}(x, \theta)=0$ and $v_{\theta}(x(\theta), \theta)=g_{\theta}(x(\theta), \theta)$. The Feynman Kac formula applied to this differential equation then yields the equation stated in the proposition.

\section{References}

Aliprantis, C., Border, K. (2006) Infinite Dimensional Analysis, Second Edition, SpringerVerlag.

Ambrosio, L., Dal Maso, G. (1990) "A General Chain Rule for Distributional Derivatives," Proceedings of the American Mathematical Society, Vol. 108, No. 3, pp. 691-702.

Bailey, P. (1968) Nonlinear Two Point Boundary Value Problems, Academic Press.

Barlow, M (1982) "One Dimensional Stochastic Differential Equations with No Strong Solution," Journal of the London Mathematical Society, Vol s2-26, pp. 335-347.

Benveniste, L. M., Scheinkman, J. A. (1979) "On the Differentiability of the Value Function in Dynamic Models of Economics," Econometrica, Vol. 47(3), pp. 727-32.

Bolton, P., Harris, C. (1999) "Strategic Experimentation," Econometrica, Vol. 67, No. 2, pp. 349-374.

Borkar, V. (2005) "Controlled Diffusion Processes," Probability Surveys, Vol. 2, pp. 213-244.

Chistyakov, V. (2004) "Selections of Bounded Variations," Journal of Applied Analysis, Vol. 10, No. 1, pp. 1-82.

Crandall, M, Lions, P.-L. (1983) "Viscosity Solutions of Hamilton-Jacobi Equations," Transactions of the American Mathematical Society, Vol. 277, No. 1, pp. 1-42.

Dayanik, S., Karatzas, I., "On the Optimal Stopping Problem for One-Dimensional Diffusions," Stochastic Processes and their Applications, Vol. 107, No. 2, pp. 173-212. 
Demarzo, P., Fishman, M., He, Z., And Wang, N. (2012) "Dynamic Agency and the $q$ Theory of Investment," forthcoming in the Journal of Finance.

DeMarzo, P., Sannikov, Y. (2006) "Optimal Security Design and Dynamic Capital Structure in a Continuous-Time Agency Model," it Journal of Finance, Vol. 61, pp. 2681-2724.

Dixit, A. (1993) The Art of Smooth Pasting, Academic Press, Harwood.

Duffie, D. (2001) Dynamic Asset Pricing Theory, Princeton University Press.

Dumas, B. (1991) "Super Contact and Related Optimality Conditions," Journal of Economic Dynamics and Control, Vol. 15, pp. 675-685.

Evans, L. C. (1983) "Classical Solutions of the Hamilton-Jacobi-Bellmann Equation of Uniformly Elliptic Operators," Transactions of the American Mathematical Society, Vol. 275, No. 1, pp. 245255.

Fleming, W. And Soner, H. (1993) Controlled Markov Processes and Viscosity Solutions, Springer Verlag, Springer.

Gilbarg, D., Trudinger, N. (2001) Elliptic Partial Differential Equations of Second Order, Springer Verlag, Springer.

Gittins, J. (1979) "Bandit Processes and Dynamic Allocation Indices," Journal of the Royal Statistical Society, Vol. 41, No. 2, pp. 148-177.

Harrison, M., Taksar, M. (1983) "Instanteneous Control of Brownian Motion," Mathematics of Operations Research, Vol. 8, No. 3, pp. 439-453.

Hartman, P. (2002) Ordinary Differential Equations, Second Edition, Classics in Applied Mathematics, SIAM.

Josephy, M. (1981) "Composing Functions of Bounded Variation," Proceedings of the American Mathematical Society, Vol. 83, pp. 354-356.

Karatzas, I., Shreve, S. (1998) Brownian Motion and Stochastic Calculus, Second Edition, Springer.

Karlin, S., Rubin, H. (1956) "The Theory of Decision Procedures for Distributions With Monotone Likelihood Ratio," Annals of Mathematical Statistics, Vol. 27, pp. 272-299.

Keller, G., Rady, S., And Cripps, M. (2005) "Strategic Experimentation with Exponential Bandits," Econometrica, Vol. 73, No. 1, pp. 39-68. 
Krylov, N. (1980) Controlled Diffusion Processes, Springer Verlag, Springer.

Lehmann, E. (1988) "Comparing Location Experiments," Annals of Statistics, Vol. 16, pp. 521533.

Leland, H. (1994) "Corporate Debt Value, Bond Covenants, and Optimal Capital Structure," Journal of Finance, Vol. 49, pp. 1213-1252.

Lions, P.-L. (1983) "Optimal Control of Diffusion Processes and Hamilton-Jacobi-Bellman Equations," Communications in Partial Differential Equations, Vol. 8, No. 11, pp. 1229-1276.

Merton, R. (1969) "Lifetime Portfolio Selection under Uncertainty: the Continuous Time Case," Review of Economic and Statistics, Vol. 51, pp. 247-257.

Merton, R. (1971) "Optimum Consumption and Portfolio Rules in a Continous Time Model," Journal of Economic Theory, Vol. 3, pp. 373-413.

Milgrom, P., Shannon, C. (1994) "Monotone Comparative Statics," Econometrica, Vol. 62, pp. $157-180$.

Milgrom, P., Segal, I. (2002) "Envelope Theorems for Arbitrary Choice Sets," Econometrica, Vol. 70(2), pp. 583-601.

NAKaO, S. (1972) "On the Pathwise Uniqueness of Solutions of One-Dimensional Stochastic Differential Equations," Osaka Journal of Mathematics, Vol. 9, pp. 513-518.

Noussair, E. S. (1979) "On the Existence of Solutions to Nonlinear Elliptic Boundary Value Problems," Journal of Differential Equations, Vol. 34, No. 3, pp. 482-495.

Øksendal, B., Sulem, A. (2007) Applied Stochastic Control of Jump Diffusions, Second Edition, Springer.

Peškir, G. AND ShIRYAev, A. (2006) Optimal Stopping and Free-Boundary Problems, Birkhauser Boston.

Pham, H. (2009) Continuous-time Stochastic Control and Optimization with Financial Applications, Springer Verlag.

Quah, J., Strulovici, B. (2009) "Comparative Statics, Informativeness, and the Interval Dominance Order," Econometrica, Vol. 77, pp. 1949-1992.

Quah, J., Strulovici, B. (2012a) "Aggregating the Single Crossing Property," forthcoming in Econometrica. 
Quah, J., Strulovici, B. (2012b) "Discounting, Values, and Decisions," Working Paper, Northwestern University and Oxford University.

Revuz, D., Yor, M. (2001) Continuous Martingales and Brownian motion, Third Edition, Springer-Verlag.

Rincón-Zapatero, J., Santos, M. (2009) "Differentiability of the value function without interiority assumptions," Journal of Economic Theory, Vol. 144(5), pp. 1948-1964.

Rincón-Zapatero, J., Santos, M. (2010) "Differentiability of the Value Function in ContinuousTime Economic Models," Working Paper.

SAfonov, M. V. (1989) "On the Classical Solution of Nonlinear Elliptic Equations of Second Order," Mathematics of the USSR-Izvestiya, Vol. 33, No. 3, pp. 597-612.

Sannikov, Y. (2008) "A Continuous-Time Version of the Principal-Agent Problem," Review of Economic Studies, Vol. 75(3), pp. 957-984.

SCHRADER, K. (1969) "Existence theorems for second order boundary value problems," Journal of Differential Equations, Vol. 5, pp. 572-584.

Stroock, D., Varadhan, S. (1979) Multidimensional Diffusion Processes, Springer.

Topkis, D. (1978) "Minimizing a Submodular Function on a Lattice," Operations Research, Vol. 26, 305-321.

Touzi, N. (2010) Deterministic and Stochastic Control, Application to Finance, Lecture Notes, Départment de Mathematiques Appliques, Ecole Polytechnique.

Veinott, A. (1989) "Lattice Programming," Unpublished Lecture Notes, Stanford University.

Veretennikov, A. (1981) "On Strong Solutions and Explicit Formulas for Solutions of Stochastic Intergal Equations," Math USSR Sb., Vol. 39, pp. 387-403.

Villeneuve, S., (2007) "On Threshold Strategies and the Smooth-Fit Principle for Optimal Stopping Problems," Journal of Applied Probability, Vol. 44, No. 1, pp. 181-198.

Yamada, T., Watanabe, S. (1971) "On the Uniqueness of Solutions of Stochastic Differential Equations," Journal of Mathematics of Kyoto University, Vol. 11, pp. 155-167.

Yong, J., Zhou, X.-Y. (1999) Stochastic Controls, Springer-Verlag. 